

\title{
On the ample cone of a rational surface with an anticanonical cycle
}

\author{
Robert Friedman
}

\begin{abstract}
Let $Y$ be a smooth rational surface, and let $D$ be a cycle of rational curves on $Y$ that is an anticanonical divisor, i.e., an element of $\left|-K_{Y}\right|$. Looijenga studied the geometry of such surfaces $Y$ in case $D$ has at most five components and identified a geometrically significant subset $R$ of the divisor classes of square -2 orthogonal to the components of $D$. Motivated by recent work of Gross, Hacking, and Keel on the global Torelli theorem for pairs $(Y, D)$, we attempt to generalize some of Looijenga's results in case $D$ has more than five components. In particular, given an integral isometry $f$ of $H^{2}(Y)$ that preserves the classes of the components of $D$, we investigate the relationship between the condition that $f$ preserves the "generic" ample cone of $Y$ and the condition that $f$ preserves the set $R$.
\end{abstract}

\section{Introduction}

The ample cone of a del Pezzo surface $Y$ (or rather the associated dual polyhedron) was studied classically by, among others, Gosset, Schoute, Kantor, Coble, Todd, Coxeter, and Du Val. For a brief historical discussion, one can consult the remarks in [Coxeter 1973, §11.x]. From this point of view, the lines on $Y$ are the main object of geometric interest as they are the walls of the ample cone or the vertices of the dual polyhedron. The corresponding root system (in case $K_{Y}^{2} \leq 6$ ) only manifests itself geometrically by allowing del Pezzo surfaces with rational double points or, equivalently, smooth surfaces $Y$ with $-K_{Y}$ nef and big but not ample. This is explicitly worked out in [Du Val 1934]. On the other hand, the root system, or rather its Weyl group, appears for a smooth del Pezzo surface as a group of symmetries of the ample cone, a fact which (in a somewhat different guise) was already known to Cartan. Perhaps the culmination of the classical side of the story is [Du Val 1937], where the blowup of $\mathbb{P}^{2}$ at $n \geq 9$ points is also systematically considered. In modern times, Manin explained the appearance of the Weyl group by noting that the orthogonal complement to $K_{Y}$ in $H^{2}(Y ; \mathbb{Z})$ is a root lattice $\Lambda$. Moreover, given any root of $\Lambda$, in other words an element $\beta$ of square -2 , there

MSC2010: $14 \mathrm{~J} 26$.

Keywords: rational surface, anticanonical cycle, exceptional curve, ample cone. 
exists a deformation of $Y$ for which $\beta= \pm[C]$, where $C$ is a smooth rational curve of self-intersection -2 . For modern expositions of the theory, see for example the book of Manin [1986] or the account of Demazure [1980a; 1980b; 1980c; 1980d].

In general, it seems hard to study an arbitrary rational surface $Y$ without imposing some extra conditions. One very natural condition is that $-K_{Y}$ is effective, i.e., that $-K_{Y}=D$ for an effective divisor $D$. In case the intersection matrix of $D$ is negative definite, such pairs $(Y, D)$ arise naturally in the study of minimally elliptic singularities: the case where $D$ is a smooth elliptic curve corresponds to the case of simple elliptic singularities, the case where $D$ is a nodal curve or a cycle of smooth rational curves meeting transversally corresponds to the case of cusp singularities, and the case where $D$ is reduced but has one component with a cusp, two components with a tacnode, or three components meeting at a point corresponds to triangle singularities. From this point of view, the case where $D$ is a cycle of rational curves is the most plentiful. The systematic study of such surfaces in case the intersection matrix of $D$ is negative definite dates back to [Looijenga 1981]. However, for various technical reasons, most of the results of that paper are proved under the assumption that the number of components in the cycle is at most 5. Some of the main points of Looijenga's seminal paper are as follows. Let $R$ denote the set of elements in $H^{2}(Y ; \mathbb{Z})$ of square -2 that are orthogonal to the components of $D$ and that are of the form $\pm[C]$, where $C$ is a smooth rational curve disjoint from $D$, for some deformation of the pair $(Y, D)$. In terms of deformations of singularities, the set $R$ is related to the possible rational double point singularities that can arise as deformations of the dual cusp to the cusp singularity corresponding to $D$. Looijenga noted that, in general, there exist elements in $H^{2}(Y ; \mathbb{Z})$ of square -2 that are orthogonal to the components of $D$ but that do not lie in $R$. Moreover, reflections in elements of the set $R$ give symmetries of the "generic" ample cone (which is the same as the ample cone in case there are no smooth rational curves on $Y$ disjoint from $D$ ). Finally, still under the assumption of at most five components, any isometry of $H^{2}(Y ; \mathbb{Z})$ that preserves the positive cone, the classes $\left[D_{i}\right]$, and the set $R$ preserves the generic ample cone.

This paper, which is an attempt to see how much of [Looijenga 1981] can be generalized to the case of arbitrarily many components, is motivated by a question raised by the recent work of Gross, Hacking, and Keel [Gross et al. 2013] on, among other matters, the global Torelli theorem for pairs $(Y, D)$ where $D$ is an anticanonical cycle on the rational surface $Y$. In order to formulate this theorem in a fairly general way, one would like to characterize the isometries $f$ of $H^{2}(Y, \mathbb{Z})$, preserving the positive cone and fixing the classes $\left[D_{i}\right]$, which preserve the ample cone of $Y$. It is natural to ask if, at least in the generic case, the condition that $f(R)=R$ is sufficient. In this paper, we give various criteria on $R$ that insure that, if an isometry $f$ of $H^{2}(Y ; \mathbb{Z})$ preserves the positive cone, the classes $\left[D_{i}\right.$ ], 
and the set $R$, then $f$ preserves the generic ample cone. Typically, one needs a hypothesis that says that $R$ is large. For example, one such hypothesis is that there is a subset of $R$ that spans a negative definite codimension-1 subspace of the orthogonal complement to the components of $D$. In theory, at least under various extra hypotheses, such a result gives a necessary and sufficient condition for an isometry to preserve the generic ample cone. In practice, however, the determination of the set $R$ in general is a difficult problem, which seems close in its complexity to the problem of describing the generic ample cone of $Y$. Finally, we show that some assumptions on $(Y, D)$ are necessary by giving examples where $R=\varnothing$, so that the condition that an isometry $f$ preserves $R$ is automatic, and of isometries $f$ such that $f$ preserves the positive cone, the classes $\left[D_{i}\right]$, and (vacuously) the set $R$ but $f$ does not preserve the generic ample cone. We do not yet have a good understanding of the relationship between preserving the ample cone and preserving the set $R$.

An outline of this paper is as follows. The preliminary Section 1 reviews standard methods for constructing nef classes on algebraic surfaces and applies this to the study of when the normal surface obtained by contracting a negative definite anticanonical cycle on a rational surface is projective. In Section 2, we analyze the ample cone and generic ample cone of a pair $(Y, D)$ and show that the set $R$ defined by Looijenga is exactly the set of elements $\beta$ in $H^{2}(Y ; \mathbb{Z})$ of square -2 that are orthogonal to the components of $D$ such that reflection about $\beta$ preserves the generic ample cone. Much of the material of Section 2 overlaps with results in [Gross et al. 2013], proved there by somewhat different methods. Section 3 is devoted to giving various sufficient conditions for an isometry $f$ of $H^{2}(Y ; \mathbb{Z})$ to preserve the generic ample cone, including the one described above. Section 4 gives examples of pairs $(Y, D)$ satisfying the sufficient conditions of Section 3 where the number of components of $D$ and the multiplicity $-D^{2}$ are arbitrarily large as well as examples showing that some hypotheses on $(Y, D)$ are necessary.

Notation and conventions. We work over $\mathbb{C}$. If $X$ is a smooth projective surface with $h^{1}\left(\mathcal{O}_{X}\right)=h^{2}\left(\mathscr{O}_{X}\right)=0$ and $\alpha \in H^{2}(X ; \mathbb{Z})$, we let $L_{\alpha}$ denote the corresponding holomorphic line bundle, i.e., $c_{1}\left(L_{\alpha}\right)=\alpha$. Given a curve $C$ or divisor class $G$ on $X$, we let $[C]$ or $[G]$ denote the corresponding element of $H^{2}(X ; \mathbb{Z})$. Intersection pairing on curves or divisors, or on elements in the second cohomology of a smooth surface (viewed as a canonically oriented 4-manifold), is denoted by multiplication.

\section{Preliminaries}

In this paper, $Y$ denotes a smooth rational surface with $-K_{Y}=D=\sum_{i=1}^{r} D_{i}$ a (reduced) cycle of rational curves; i.e., each $D_{i}$ is a smooth rational curve and $D_{i}$ meets $D_{i \pm 1}$ transversally, where $i$ is taken $\bmod r$ except for $r=1$, in which case $D_{1}=D$ is an irreducible nodal curve. We note, however, that many of the results 
in this paper can be generalized to the case where $D \in\left|-K_{Y}\right|$ is not assumed to be a cycle. The integer $r=r(D)$ is called the length of $D$. An orientation of $D$ is an orientation of the dual graph (with appropriate modifications in case $r=1$ ). We shall abbreviate the data of the surface $Y$ and the oriented cycle $D$ by $(Y, D)$ and refer to it as an anticanonical pair. If the intersection matrix $\left(D_{i} \cdot D_{j}\right)$ is negative definite, we say that $(Y, D)$ is a negative definite anticanonical pair.

Definition 1.1. An irreducible curve $E$ on $Y$ is an exceptional curve if $E \cong \mathbb{P}^{1}$, $E^{2}=-1$, and $E \neq D_{i}$ for any $i$. An irreducible curve $C$ on $Y$ is a-2-curve if $C \cong \mathbb{P}^{1}, C^{2}=-2$, and $C \neq D_{i}$ for any $i$. Let $\Delta_{Y}$ be the set of all-2-curves on $Y$, and let $\mathrm{W}\left(\Delta_{Y}\right)$ be the group of integral isometries of $H^{2}(Y ; \mathbb{R})$ generated by the reflections in the classes in the set $\Delta_{Y}$.

Definition 1.2. Let $\Lambda=\Lambda(Y, D) \subseteq H^{2}(Y ; \mathbb{Z})$ be the orthogonal complement of the lattice spanned by the classes $\left[D_{i}\right]$. Fixing the identification $\operatorname{Pic}^{0} D \cong \mathbb{G}_{m}$ defined by the orientation of the cycle $D$, we define the period homomorphism $\varphi_{Y}: \Lambda \rightarrow \mathbb{G}_{m}$ as follows: if $\alpha \in \Lambda$ and $L_{\alpha}$ is the corresponding line bundle, then $\varphi_{Y}(\alpha) \in \mathbb{G}_{m}$ is the image of the line bundle of multidegree 0 on $D$ defined by $L_{\alpha} \mid D$. Clearly $\varphi_{Y}$ is a homomorphism. The period map is the function that associates to the pair $(Y, D)$ the homomorphism $\varphi_{Y}: \Lambda \rightarrow \mathbb{G}_{m}$.

By [Looijenga 1981; Friedman and Scattone 1986; Friedman 1984], we have:

Theorem 1.3. The period map is surjective. More precisely, given $Y$ as above and given an arbitrary homomorphism $\varphi: \Lambda \rightarrow \mathbb{G}_{m}$, there exists a deformation of the pair $(Y, D)$ over a smooth connected base, which we can take to be $\left(\mathbb{G}_{m}\right)^{n}$ for some $n$, such that the monodromy of the family is trivial and there exists a fiber of the deformation, say $\left(Y^{\prime}, D^{\prime}\right)$, such that $\varphi_{Y^{\prime}}=\varphi$ under the induced identification of $\Lambda\left(Y^{\prime}, D^{\prime}\right)$ with $\Lambda$.

For future reference, we recall some standard facts about negative definite curves on a surface.

Lemma 1.4. Let $X$ be a smooth projective surface, and let $G_{1}, \ldots, G_{n}$ be irreducible curves on $X$ such that the intersection matrix $\left(G_{i} \cdot G_{j}\right)$ is negative definite. Let $F$ be an effective divisor on $X$ not necessarily reduced or irreducible and such that, for all $i, G_{i}$ is not a component of $F$.

(i) Given $r_{i} \in \mathbb{R}$, if $\left(F+\sum_{i} r_{i} G_{i}\right) \cdot G_{j}=0$ for all $j$, then $r_{i} \geq 0$ for all $i$, and, for every subset I of $\{1, \ldots, n\}$, if $\bigcup_{i \in I} G_{i}$ is a connected curve such that $F \cdot G_{j} \neq 0$ for some $j \in I$, then $r_{i}>0$ for $i \in I$.

(ii) Given $s_{i}, t_{i} \in \mathbb{R}$, if $[F]+\sum_{i} s_{i}\left[G_{i}\right]=\sum_{i} t_{i}\left[G_{i}\right]$, then $F=0$ and $s_{i}=t_{i}$ for all $i$.

The following general result is also well known: 
Proposition 1.5. Let $X$ be a smooth projective surface, and let $G_{1}, \ldots, G_{n}$ be irreducible curves on $X$ such that the intersection matrix $\left(G_{i} \cdot G_{j}\right)$ is negative definite. (We do not, however, assume that $\bigcup_{i} G_{i}$ is connected.) Then there exists a nef and big divisor $H$ on $X$ such that $H \cdot G_{j}=0$ for all $j$ and, if $C$ is an irreducible curve such that $C \neq G_{j}$ for any $j$, then $H \cdot C>0$. In fact, the set of nef and big $\mathbb{R}$-divisors that are orthogonal to $\left\{G_{1}, \ldots, G_{n}\right\}$ is a nonempty open subset of $\left\{G_{1}, \ldots, G_{n}\right\}^{\perp} \otimes \mathbb{R}$.

Proof. Fix an ample divisor $H_{0}$ on $X$. Since $\left(G_{i} \cdot G_{j}\right)$ is negative definite, there exist $r_{i} \in \mathbb{Q}$ such that $\left(\sum_{i} r_{i} G_{i}\right) \cdot G_{j}=-\left(H_{0} \cdot G_{j}\right)$ for every $j$. Hence, $\left(H_{0}+\sum_{i} r_{i} G_{i}\right) \cdot G_{j}=0$. By Lemma $1.4, r_{i}>0$ for every $i$. There exists an $N>0$ such that $N r_{i} \in \mathbb{Z}$ for all $i$. Then $H=N\left(H_{0}+\sum_{i} r_{i} G_{i}\right)$ is an effective divisor satisfying $H \cdot G_{j}=0$ for all $j$. If $C$ is an irreducible curve such that $C \neq G_{j}$ for any $j$, then $H_{0} \cdot C>0$ and $G_{i} \cdot C \geq 0$ for all $i$. Hence, $H \cdot C>0$. In particular, $H$ is nef. Finally, $H$ is big since $H^{2}=N H \cdot\left(H_{0}+\sum_{i} r_{i} G_{i}\right)=N\left(H \cdot H_{0}\right)>0$ as $H_{0}$ is ample.

To see the final statement, we apply the above argument to an ample $\mathbb{R}$-divisor $x$ (i.e., an element in the interior of the ample cone) to see that $x+\sum_{i} r_{i} G_{i}$ is a nef and big $\mathbb{R}$-divisor orthogonal to $\left\{G_{1}, \ldots, G_{n}\right\}$. As $x+\sum_{i} r_{i} G_{i}$ is simply the orthogonal projection $p$ of $x$ onto $\left\{G_{1}, \ldots, G_{n}\right\}^{\perp} \otimes \mathbb{R}$ and $p: H^{2}(X ; \mathbb{R}) \rightarrow\left\{G_{1}, \ldots, G_{n}\right\}^{\perp} \otimes \mathbb{R}$ is an open map, the image of the interior of the ample cone of $X$ is then a nonempty open subset of $\left\{G_{1}, \ldots, G_{n}\right\}^{\perp} \otimes \mathbb{R}$ consisting of nef and big $\mathbb{R}$-divisors orthogonal to $\left\{G_{1}, \ldots, G_{n}\right\}$.

Applying the above construction to $X=Y$ and $D_{1}, \ldots, D_{r}$, we can find a nef and big divisor $H$ such that $H \cdot D_{j}=0$ for all $j$ and such that, if $C$ is an irreducible curve such that $C \neq D_{j}$ for any $j$, then $H \cdot C>0$.

Proposition 1.6. Let $(Y, D)$ be a negative definite anticanonical pair, and let $H$ be a nef and big divisor such that $H \cdot D_{j}=0$ for all $j$ and such that, if $C$ is an irreducible curve such that $C \neq D_{j}$ for any $j$, then $H \cdot C>0$. Suppose in addition that $O_{Y}(H) \mid D=O_{D}$, i.e., that $\varphi_{Y}([H])=1$. Then the $D_{i}$ are not fixed components of $|H|$. Hence, if $\bar{Y}$ denotes the normal complex surface obtained by contracting the $D_{i}$, then $H$ induces an ample divisor $\bar{H}$ on $\bar{Y}$ and $|3 \bar{H}|$ defines an embedding of $\bar{Y}$ in $\mathbb{P}^{N}$ for some $N$.

Proof. Consider the exact sequence

$$
0 \rightarrow \mathrm{O}_{Y}(H-D) \rightarrow \mathrm{O}_{Y}(H) \rightarrow \mathrm{O}_{D} \rightarrow 0 .
$$

Looking at the long exact cohomology sequence, as

$$
H^{1}\left(Y ; \mathscr{O}_{Y}(H-D)\right)=H^{1}\left(Y ; \mathscr{O}_{Y}(H) \otimes K_{Y}\right)
$$

is Serre dual to $H^{1}\left(Y ; \mathscr{O}_{Y}(-H)\right)=0$, by Ramanujam's vanishing theorem, there exists a section of $\mathrm{O}_{Y}(H)$ that is nowhere vanishing on $D$, proving the first statement. 
The second follows from the Nakai-Moishezon criterion and the third from general results on linear series on anticanonical pairs [Friedman 1983].

Remark 1.7. By the surjectivity of the period map (Theorem 1.3), for any $(Y, D)$ a negative definite anticanonical pair and $H$ a nef and big divisor on $Y$ such that $H \cdot D_{j}=0$ for all $j$ and $H \cdot C>0$ for all curves $C \neq D_{i}$, there exists a deformation of the pair $(Y, D)$ such that the divisor corresponding to $H$ has trivial restriction to $D$. More generally, one can consider deformations such that $\varphi_{Y}([H])$ is a torsion point of $\mathbb{G}_{m}$. In this case, if $\bar{Y}$ is the normal surface obtained by contracting $D$, then $\bar{Y}$ is projective. Note that this implies that the set of pairs $(Y, D)$ such that $\bar{Y}$ is projective is Zariski dense in the moduli space. However, as the set of torsion points is not dense in $\mathbb{G}_{m}$ in the classical topology, the set of projective surfaces $\bar{Y}$ will not be dense in the classical topology.

\section{Roots and nodal classes}

Definition 2.1. Let $\mathscr{b}=\mathscr{C}(Y)$ be the positive cone of $Y$, i.e.,

$$
\mathscr{C}=\left\{x \in H^{2}(Y ; \mathbb{R}): x^{2}>0\right\} .
$$

Then $\mathscr{C}$ has two components, and exactly one of them, say $\mathscr{C}^{+}=\mathscr{C}^{+}(Y)$, contains the classes of ample divisors. We also define

$$
\mathscr{C}_{D}^{+}=\mathscr{C}_{D}^{+}(Y)=\left\{x \in \mathscr{C}^{+}: x \cdot\left[D_{i}\right] \geq 0 \text { for all } i\right\} .
$$

Let $\bar{A}(Y) \subseteq \mathscr{C}^{+} \subseteq H^{2}(Y ; \mathbb{R})$ be (the closure of) the ample (nef, Kähler) cone of $Y$ in $\mathscr{C}^{+}$. By definition, $\bar{A}(Y)$ is closed in $\mathscr{C}^{+}$but not in general in $H^{2}(Y ; \mathbb{R})$.

Definition 2.2. Let $\alpha \in H^{2}(Y ; \mathbb{Z}), \alpha \neq 0$. The oriented wall $W^{\alpha}$ associated to $\alpha$ is the set $\left\{x \in \mathscr{C}^{+}: x \cdot \alpha=0\right\}$, i.e., the intersection of $\mathscr{C}^{+}$with the orthogonal space to $\alpha$ together with the preferred half space defined by $x \cdot \alpha \geq 0$. If $C$ is a curve on $Y$, we write $W^{C}$ for $W^{[C]}$. A standard result (see, for example, [Friedman and Morgan 1988 , II (1.8)]) shows that, if $I$ is a subset of $H^{2}(Y ; \mathbb{Z})$ and there exists an $N \in \mathbb{Z}^{+}$ such that $-N \leq \alpha^{2}<0$ for all $\alpha \in I$, then the collection of walls $\left\{W^{\alpha}: \alpha \in I\right\}$ is locally finite on $\mathscr{C}^{+}$. Finally, we say that $W^{\alpha}$ is a face of $\bar{A}(Y)$ if $\partial \bar{A}(Y) \cap W^{\alpha}$ contains a nonempty open subset of $W^{\alpha}$ and $x \cdot \alpha \geq 0$ for all $x \in \bar{A}(Y)$.

Lemma 2.3. $\bar{A}(Y)$ is the set of all $x \in \mathscr{C}^{+}$such that $x \cdot\left[D_{i}\right] \geq 0, x \cdot[E] \geq 0$ for all exceptional curves $E$, and $x \cdot[C] \geq 0$ for all-2-curves $C$. Moreover, if $\alpha$ is the class associated to an exceptional or -2-curve, or $\alpha=\left[D_{i}\right]$ for some $i$ such that $D_{i}^{2}<0$, then $W^{\alpha}$ is a face of $\bar{A}(Y)$. If $\alpha$ and $\beta$ are two such classes, $W^{\alpha}=W^{\beta} \Longleftrightarrow \alpha=\beta$.

Proof. For the first claim, it is enough to show that, if $G$ is an irreducible curve on $Y$ with $G^{2}<0$, then $G$ is either $D_{i}$ for some $i$, an exceptional curve, or a -2-curve. This follows immediately from adjunction since, if $G \neq D_{i}$ for any $i$, then $G \cdot D \geq 0$ 
and $-2 \leq 2 p_{a}(G)-2=G^{2}-G \cdot D<0$; hence, $p_{a}(G)=0$ and either $G^{2}=-2$, $G \cdot D=0$, or $G^{2}=G \cdot D=-1$. The last two statements follow from the openness statement in Proposition 1.5 and the fact that no two distinct classes of the types listed above are multiples of each other.

As an alternate characterization of the classes in the previous lemma, we have the following:

Lemma 2.4. Let $H$ be a nef divisor such that $H \cdot D>0$.

(i) If $\alpha \in H^{2}(Y ; \mathbb{Z})$ with $\alpha^{2}=\alpha \cdot\left[K_{Y}\right]=-1$, then $\alpha \cdot[H] \geq 0$ if and only if $\alpha$ is the class of an effective curve. In particular, the wall $W^{\alpha}$ does not pass through the interior of $\bar{A}(Y)$. (See [Friedman and Morgan 1988, p. 332] for a more general statement.)

(ii) If $\beta \in H^{2}(Y ; \mathbb{Z})$ with $\beta^{2}=-2, \beta \cdot\left[D_{i}\right]=0$ for all $i, \beta \cdot[H] \geq 0$, and $\varphi_{Y}(\beta)=1$, then $\pm \beta$ is the class of an effective curve, and $\beta$ is effective if $\beta \cdot[H]>0$.

Hence, the ample cone $\bar{A}(Y)$ is the set of all $x \in \mathscr{C}^{+}$such that $x \cdot\left[D_{i}\right] \geq 0$ and $x \cdot \alpha \geq 0$ for all classes $\alpha$ and $\beta$ as described in (i) and (ii) above, where in case (ii) we assume in addition that $\beta$ is effective or equivalently that $\beta \cdot[H]>0$ for some nef divisor $H$.

Proof. (i) Clearly, if $\alpha$ is the class of an effective curve, then $\alpha \cdot[H] \geq 0$ since $H$ is nef. Conversely, assume that $\alpha^{2}=\alpha \cdot\left[K_{Y}\right]=-1$ and that $\alpha \cdot[H] \geq 0$. By the Riemann-Roch theorem, $\chi\left(L_{\alpha}\right)=1$. Hence, either $h^{0}\left(L_{\alpha}\right)>0$ or $h^{2}\left(L_{\alpha}\right)>0$. But $h^{2}\left(L_{\alpha}\right)=h^{0}\left(L_{\alpha}^{-1} \otimes K_{Y}\right)$ and $[H] \cdot(-\alpha-[D])<0$ by assumption. Thus, $h^{0}\left(L_{\alpha}\right)>0$ and hence $\alpha$ is the class of an effective curve.

(ii) As in (i), $H \cdot(-\beta-[D])<0$, and hence, $h^{0}\left(L_{\beta}^{-1} \otimes K_{Y}\right)=0$. Thus, $h^{2}\left(L_{\beta}\right)=0$. Suppose that $h^{0}\left(L_{\beta}\right)=0$. Then, by the Riemann-Roch theorem, $\chi\left(L_{\beta}\right)=0$ and hence $h^{1}\left(L_{\beta}\right)=0$. Hence, $h^{1}\left(L_{\beta}^{-1} \otimes K_{Y}\right)=0$. Since $\varphi_{Y}(\beta)=1, L_{\beta}^{ \pm 1} \mid D=O_{D}$. Thus, there is an exact sequence

$$
0 \rightarrow L_{\beta}^{-1} \otimes \mathrm{O}_{Y}(-D) \rightarrow L_{\beta}^{-1} \rightarrow \mathrm{O}_{D} \rightarrow 0 .
$$

Since $H^{1}\left(L_{\beta}^{-1} \otimes K_{Y}\right)=H^{1}\left(L_{\beta}^{-1} \otimes \mathscr{O}_{Y}(-D)\right)=0$, the map $H^{0}\left(L_{\beta}^{-1}\right) \rightarrow H^{0}\left(\mathscr{O}_{D}\right)$ is surjective and hence $-\beta$ is the class of an effective curve.

Definition 2.5. Let $\alpha \in H^{2}(Y ; \mathbb{Z})$. Then $\alpha$ is a numerical exceptional curve if $\alpha^{2}=\alpha \cdot\left[K_{Y}\right]=-1$. The numerical exceptional curve $\alpha$ is effective if $h^{0}\left(L_{\alpha}\right)>0$, i.e., if $\alpha=[G]$, where $G$ is an effective curve.

A minor variation of the proof of Lemma 2.4 shows the following:

Lemma 2.6. Let $H$ be a nef and big divisor such that $H \cdot G>0$ for all irreducible curves $G$ not equal to $D_{i}$ for some $i$, and let $\alpha$ be a numerical exceptional curve. 
(i) Suppose that $[H] \cdot \alpha \geq 0$. Then either $[H] \cdot \alpha>0$ and $\alpha$ is effective or $H \cdot D=[H] \cdot \alpha=0$ and $\alpha$ is an integral linear combination of the $\left[D_{i}\right]$.

(ii) If $(Y, D)$ is negative definite and $\alpha$ is an integral linear combination of the $\left[D_{i}\right]$, then either some component $D_{i}$ is a smooth rational curve of selfintersection -1 or $K_{Y}^{2}=-1, \alpha=K_{Y}$, and hence $\alpha$ is not effective.

(iii) If no component $D_{i}$ is a smooth rational curve of self-intersection -1 , then $\alpha$ is effective if and only if $[H] \cdot \alpha>0$.

Proof. (i) As in the proof of Lemma 2.4, either $\alpha$ or $-\alpha-[D]$ is the class of an effective divisor. If $-\alpha-[D]$ is the class of an effective divisor, then $0 \leq[H] \cdot(-\alpha-[D]) \leq 0$, so $[H] \cdot \alpha=H \cdot D=0$. In particular, $(Y, D)$ is negative definite. Moreover, if $G$ is an effective divisor with $[G]=-\alpha-[D]$, then every component of $G$ is equal to some $D_{i}$. Hence, $[G]$ and therefore $\alpha=-[G]-[D]$ are integral linear combinations of the $\left[D_{i}\right]$.

(ii) Suppose that $\alpha$ is an integral linear combination of the $\left[D_{i}\right]$ but that no $D_{i}$ is a smooth rational curve of self-intersection -1 . We shall show that $K_{Y}^{2}=-1$ and $\alpha=K_{Y}$. First suppose that $K_{Y}^{2}=-1$. Then $\bigoplus_{i} \mathbb{Z} \cdot\left[D_{i}\right]=\mathbb{Z} \cdot\left[K_{Y}\right] \oplus L$, where $L$, the orthogonal complement of $\left[K_{Y}\right]$ in $\bigoplus_{i} \mathbb{Z} \cdot\left[D_{i}\right]$, is even and negative definite. Thus, $\alpha=a\left[K_{Y}\right]+\beta$, with either $\beta=0$ or $\beta^{2} \leq-2$, and $\alpha^{2}=-a^{2}+\beta^{2}$. Hence, if $\alpha^{2}=\alpha \cdot\left[K_{Y}\right]=-1$, the only possibility is $\beta=0$ and $a=1$. In case $K_{Y}^{2}<-1$, $D$ is reducible, and no $D_{i}$ is a smooth rational curve of self-intersection -1 , then $D_{i}^{2} \leq-2$ for all $i$ and either $D_{i}^{2} \leq-4$ for some $i$ or there exist $i \neq j$ such that $D_{i}^{2}=D_{j}^{2}=-3$. In this case, it is easy to check that, for all integers $a_{i}$ such that $a_{i} \neq 0$ for some $i,\left(\sum_{i} a_{i} D_{i}\right)^{2}<-1$. This contradicts $\alpha^{2}=-1$.

(iii) If [H] $\alpha>0$, then $\alpha$ is effective by (i). If [H] $\alpha<0$, then clearly $\alpha$ is not effective. Suppose that $[H] \cdot \alpha=0$; we must show that, again, $\alpha$ is not effective. Suppose that $\alpha=[G]$ is effective. By the hypothesis on $H$, every component of $G$ is a $D_{i}$ for some $i$ so that $\alpha=\sum_{i} a_{i}\left[D_{i}\right]$ for some $a_{i} \in \mathbb{Z}, a_{i} \geq 0$. Let $I \subseteq\{1, \ldots, r\}$ be the set of $i$ such that $a_{i}>0$. Then $H \cdot D_{i}=0$ for all $i \in I$. If $I=\{1, \ldots, r\}$, then $(Y, D)$ is negative definite and we are done by (ii). Otherwise, $\bigcup_{i \in I} D_{i}$ is a union of chains of curves whose components $D_{i}$ satisfy $D_{i}^{2} \leq-2$. It is then easy to check that $\alpha^{2}<-1$ in this case, a contradiction. Hence, $\alpha$ is not effective.

Definition 2.7. Let $Y_{t}$ be a generic small deformation of $Y$, and identify $H^{2}\left(Y_{t} ; \mathbb{R}\right)$ with $H^{2}(Y ; \mathbb{R})$. Define $\bar{A}_{\text {gen }}=\bar{A}_{\text {gen }}(Y)$ to be the ample cone $\bar{A}\left(Y_{t}\right)$ of $Y_{t}$, viewed as a subset of $H^{2}(Y ; \mathbb{R})$.

Lemma 2.8. With notation as above, the following are true:

(i) If there do not exist any-2-curves on $Y$, then $\bar{A}(Y)=\bar{A}_{\text {gen }}$. More generally, $\bar{A}_{\text {gen }}$ is the set of all $x \in \mathscr{C}^{+}$such that $x \cdot\left[D_{i}\right] \geq 0$ and $x \cdot \alpha \geq 0$ for all effective numerical exceptional curves. In particular, $\overline{\mathscr{A}}(Y) \subseteq \overline{\mathscr{A}}_{\text {gen }}$. 
(ii) We have $\bar{A}(Y)=\left\{x \in \bar{A}_{\text {gen }}: x \cdot[C] \geq 0\right.$ for all-2-curves $\left.C\right\}$.

Proof. Let $Y$ be a surface with no -2-curves (such surfaces exist and are generic by the surjectivity of the period map (Theorem 1.3)). Fix a nef divisor $H$ on $Y$ with $H \cdot D>0$. Then $\bar{A}(Y)$ is the set of all $x \in \mathscr{C}^{+}$such that $x \cdot\left[D_{i}\right] \geq 0$ and $x \cdot[E] \geq 0$ for all exceptional curves $E$, and this last condition is equivalent to $x \cdot \alpha \geq 0$ for all $\alpha \in H^{2}(Y ; \mathbb{Z})$ such that $\alpha^{2}=\alpha \cdot\left[K_{Y}\right]=-1$ and $\alpha \cdot[H] \geq 0$ by Lemma 2.4. Since this condition is independent of the choice of $Y$, because we can choose the divisor $H$ to be ample and to vary in a small deformation, the first part of (i) follows, and the remaining statements are clear.

In fact, the argument above shows the following:

Lemma 2.9. The set of effective numerical exceptional curves and the set $\overline{\mathscr{A}}_{\text {gen }}$ are locally constant and hence are invariant in a global deformation with trivial monodromy under the induced identifications.

Lemma 2.10. If $C$ is a-2-curve on $Y$, then the wall $W^{C}$ meets the interior of $\overline{\mathscr{A}}_{\text {gen }}$, and in fact, $r_{C}\left(\overline{\mathscr{A}}_{\mathrm{gen}}\right)=\overline{\mathscr{A}}_{\mathrm{gen}}$, where $r_{C}: H^{2}(Y ; \mathbb{R}) \rightarrow H^{2}(Y ; \mathbb{R})$ is reflection in the class $[C]$. Hence, $\overline{\mathcal{A}}(Y)$ is a fundamental domain for the action of the group $\mathrm{W}\left(\Delta_{Y}\right)$ on $\bar{A}_{\text {gen }}$, where $\mathrm{W}\left(\Delta_{Y}\right)$ is the group generated by the reflections in the classes in the set $\Delta_{Y}$ of-2-curves on $Y$.

Proof. Clearly, if $r_{C}\left(\overline{\mathscr{A}}_{\text {gen }}\right)=\overline{\mathscr{A}}_{\text {gen }}$, then $W^{C}$ meets the interior of $\overline{\mathscr{A}}_{\text {gen }}$. To see that $r_{C}\left(\overline{\mathscr{A}}_{\text {gen }}\right)=\overline{\mathscr{A}}_{\text {gen }}$, assume first more generally that $\beta \in \Lambda$ is any class with $\beta^{2}=-2$, and let $r_{\beta}$ be the corresponding reflection. Then $r_{\beta}$ permutes the set of $\alpha \in H^{2}(Y ; \mathbb{Z})$ such that $\alpha^{2}=\alpha \cdot\left[K_{Y}\right]=-1$ but does not necessarily preserve the condition that $\alpha$ is effective, i.e., that $\alpha \cdot[H] \geq 0$ for some nef divisor $H$ on $Y$ with $H \cdot D>0$. However, for $\beta=[C]$, there exists by Proposition 1.5 a nef and big divisor $H_{0}$ such that $H_{0} \cdot C=0$ and $H \cdot D>0$. Hence, $\left[H_{0}\right]$ is invariant under $r_{C}$, and so $r_{C}$ permutes the set of $\alpha \in H^{2}(Y ; \mathbb{Z})$ such that $\alpha^{2}=\alpha \cdot\left[K_{Y}\right]=-1$ and $\alpha \cdot\left[H_{0}\right] \geq 0$. Thus, $r_{C}$ permutes the set of effective numerical exceptional curves and hence the faces of $\bar{A}_{\text {gen }}$ so that $r_{C}\left(\bar{A}_{\text {gen }}\right)=\overline{\mathscr{A}}_{\text {gen }}$. Since $\bar{A}(Y) \subseteq \overline{\mathscr{A}}_{\text {gen }}$ is given by Lemma 2.8(ii), the final statement is then a general result in the theory of reflection groups [Bourbaki 1981, V §3].

Remark 2.11. (i) The argument for the first part of Lemma 2.10 essentially boils down to the following. Let $\bar{Y}$ be the normal surface obtained by contracting $C$. Then the reflection $r_{C}$ is the monodromy associated to a generic smoothing of the singular surface $\bar{Y}$, and the cone $\overline{\mathcal{A}}_{\text {gen }}$ is invariant under monodromy.

(ii) If $E$ is an exceptional curve, then $W^{E}$ is a face of $\bar{A}(Y)$. For a generic $Y$ (i.e., no -2-curves), Lemma 2.10 then says that the set of exceptional curves on $Y$ is invariant under the reflection group generated by all classes of square -2 that become the classes of a -2-curve under some specialization. A somewhat more involved statement holds in the nongeneric case. 
Lemma 2.12. With $\mathrm{W}\left(\Delta_{Y}\right)$ as in Definition 1.1, for all $w \in \mathrm{W}\left(\Delta_{Y}\right)$ and all $\beta \in \Lambda$, $\varphi_{Y}(w(\alpha))=\varphi_{Y}(\alpha)$.

Proof. This is clear since $\varphi_{Y}([C])=1$ implies $\varphi_{Y}\left(r_{C}(\alpha)\right)=\varphi_{Y}(\alpha)$ for all $\alpha \in \Lambda$.

Lemma 2.13. Suppose that $C=\sum_{i} a_{i} C_{i}$, where the $C_{i}$ are -2-curves, $a_{i} \in \mathbb{Z}$, $C^{2}=-2$, the support of $C$ is connected, and $\left(C_{i} \cdot C_{j}\right)$ is negative definite. Then there exists an element $w$ in the group generated by reflections in the $\left[C_{i}\right]$ such that $w([C])=\left[C_{i}\right]$ for some $i$.

Proof. This follows from the well known fact that, if $R$ is an irreducible root system such that all roots have the same length, then the Weyl group W $(R)$ acts transitively on the set of roots.

Theorem 2.14. Let $\beta \in \Lambda$ with $\beta^{2}=-2$. Then the following are equivalent:

(i) Let $Y_{1}$ be a deformation of $Y$ with trivial monodromy such that $\varphi_{Y_{1}}(\beta)=1$. Then, with $\mathrm{W}\left(\Delta_{Y_{1}}\right)$ as in Definition 1.1, there exists $w \in \mathrm{W}\left(\Delta_{Y_{1}}\right)$ such that $w(\beta)=[C]$, where $C$ is a-2-curve on $Y_{1}$. In particular, if $Y_{1}$ is generic subject to the condition that $\varphi_{Y_{1}}(\beta)=1$ (i.e., if $\operatorname{Ker} \varphi_{Y_{1}}=\mathbb{Z} \cdot \beta$ ), then $\pm \beta=[C]$ for a-2-curve $C$.

(ii) The wall $W^{\beta}$ meets the interior of $\bar{A}_{\mathrm{gen}}$.

(iii) If $r_{\beta}$ is reflection in the class $\beta$, then $r_{\beta}\left(\bar{A}_{\mathrm{gen}}\right)=\overline{\mathscr{A}}_{\mathrm{gen}}$.

Proof. Lemma 2.10 implies that (i) $\Longrightarrow$ (iii) in case $Y=Y_{1}$ and $\beta=[C]$ where $C$ is a -2 -curve. The case where $w(\beta)=[C]$ follows easily from this since, for all $w \in \mathrm{W}\left(\Delta_{Y_{1}}\right), w \circ r_{\beta} \circ w^{-1}=r_{w(\beta)}$. Lemma 2.9 then handles the case where $Y_{1}$ is replaced by a general deformation $Y$. Also, clearly (iii) $\Longrightarrow$ (ii). So it is enough to show that (ii) $\Longrightarrow$ (i). In fact, by Lemma 2.13 , it is enough to show that, if $Y$ is any surface such that $\varphi_{Y}(\beta)=1$ and $W^{\beta}$ meets the interior of $\bar{A}_{\text {gen }}$, then there exists a $w \in \mathrm{W}\left(\Delta_{Y}\right)$ such that $w(\beta)=\left[\sum_{i} a_{i} C_{i}\right]$ where $a_{i} \in \mathbb{Z}^{+}$, the $C_{i}$ are curves disjoint from $D$, and $\bigcup_{i} C_{i}$ is connected.

By hypothesis, there exists an $x$ in the interior of $\bar{A}_{\text {gen }}$ such that $x \cdot \beta=0$. In particular, $x \cdot\left[D_{i}\right]>0$ for all $i$. We can assume that $x=[H]$ is the class of a divisor $H$. After replacing $x$ by $w(x)$ and $\beta$ by $w(\beta)$ for some $w \in \mathrm{W}\left(\Delta_{Y}\right)$, we can assume that $x$ (and hence $H$ ) lies in $\bar{A}(Y)$ so that $H$ is a nef and big divisor with $H \cdot D_{i}>0$ for all $i$, and we still have $\varphi_{Y}(\beta)=1$ by Lemma 2.12. By Lemma 2.4, possibly after replacing $\beta$ by $-\beta, \beta=\left[\sum_{i} a_{i} C_{i}\right]$ where the $C_{i}$ are irreducible curves and $a_{i} \in \mathbb{Z}^{+}$. Since $\beta \cdot[H]=\sum_{i} a_{i}\left(C_{i} \cdot H\right)=0, C_{i} \cdot H \geq 0$, and $D_{j} \cdot H>0$, $C_{i} \cdot H=0$ for all $i$, and no $C_{i}$ is equal to $D_{j}$ for any $j$. Hence, the $C_{i}$ are curves meeting each $D_{j}$ in at most finitely many points and $\sum_{i} a_{i}\left(C_{i} \cdot D_{j}\right)=0$ so that $C_{i} \cap D_{j}=\varnothing$. Finally, each $\left(C_{i}\right)^{2}<0$ by Hodge index, and so each $C_{i}$ is a -2 -curve. Moreover, the $C_{i}$ span a negative definite lattice, and in particular, their classes are independent. From this, the statement about the connectedness of $\bigcup_{i} C_{i}$ is clear. 
Definition 2.15. Let $R=R_{Y}$ be the set of all $\beta \in \Lambda$ such that $\beta^{2}=-2$ and such that there exists some deformation of $Y$ for which $\beta$ becomes the class of a -2-curve. Following [Gross et al. 2013], we call $R$ the set of Looijenga roots (or briefly roots) of $Y$. Note that $R$ only depends on the deformation type of $Y$.

The definition of $R$ is slightly ill-posed since we have not specified an identification of the cohomologies of the fibers along the deformation. In particular, if $\beta=[C]$ is a -2 -curve on $Y$, then by Remark 2.11(i) if $Y^{\prime}$ is a nearby deformation of $Y$, then a general smoothing of the ordinary double point on the contraction of $C$ on $Y$ has monodromy that sends $[C]$ to $-[C]$, and hence, $-\beta \in R$ as well. To avoid this issue, it is simpler to define $R$ to be the set of $\beta \in \Lambda, \beta^{2}=-2$, which satisfy either of the equivalent conditions Theorem 2.14(ii)-(iii).

Given $Y$, let $\Delta_{Y}$ be the set of classes of -2-curves on $Y$ and $\mathrm{W}\left(\Delta_{Y}\right)$ the reflection group generated by $\Delta_{Y}$. Finally set $R^{\text {nod }}$, the set of nodal classes, to be $\mathrm{W}\left(\Delta_{Y}\right) \cdot \Delta_{Y}$. Then $R^{\text {nod }} \subseteq R$.

Corollary 2.16. (i) If $f: H^{2}(Y ; \mathbb{Z}) \rightarrow H^{2}(Y$; $\mathbb{Z})$ is an integral isometry preserving the classes $\left[D_{i}\right]$ such that $f\left(\overline{\mathscr{A}}_{\mathrm{gen}}\right)=\overline{\mathscr{A}}_{\mathrm{gen}}$, then $f(R)=R$.

(ii) If $\mathrm{W}(R)$ is the reflection group generated by reflections in the elements of $R$, then $\mathrm{W}(R) \cdot R=R$ and $w\left(\bar{A}_{\text {gen }}\right)=\overline{\mathscr{A}}_{\text {gen }}$ for all $w \in \mathrm{W}(R)$.

Remark 2.17. A result similar to Theorem 2.14 classifies the elements of $H^{2}(Y ; \mathbb{Z})$ that are represented by the class of a smoothly embedded 2-sphere of self-intersection -2 in terms of the "super $P$-cell" of [Friedman and Morgan 1988].

For the case where the length $r(D) \leq 5$, Looijenga [1981] defines a subset $R_{L}$ of $\Lambda$ by starting with a particular configuration $B$ of elements of square -2 (a root basis in his terminology) and setting $R_{L}=\mathrm{W}(B) \cdot B$, where $\mathrm{W}(B)$ is the reflection group generated by $B$. In fact, the set $R_{L}$ is just the set $R$ of Looijenga roots.

Proposition 2.18. In the above notation, $R_{L}=R$.

Proof. It is easy to see from the construction of [Looijenga 1981, I §2] that $B \subseteq R$. Hence, $R_{L} \subseteq R$. Conversely, if $\alpha \in R$, then, by Corollary $2.16($ ii $), r_{\alpha}\left(\overline{\mathscr{A}}_{\text {gen }}\right)=\overline{\mathscr{A}}_{\text {gen }}$. It then follows from [Looijenga 1981, Proposition I (4.7)] that $r_{\alpha} \in \mathrm{W}(B)$. By a general result in the theory of reflection groups [Bourbaki 1981, V §3.2, Theorem 1(iv)], $r_{\alpha}=r_{\beta}$ for some $\beta \in R_{L}$. Thus, $\alpha= \pm \beta$ so that $\alpha \in R_{L}$. Hence, $R \subseteq R_{L}$, and therefore, $R_{L}=R$.

Example 2.19. Let $(Y, D)$ be the blowup of $\mathbb{P}^{2}$ at $N \geq 10$ general points on an irreducible nodal cubic curve. We let $h$ be the pullback of the class of a line on $\mathbb{P}^{2}$ and $e_{1}, \ldots, e_{N}$ be the classes of the exceptional curves.

(i) Let $\alpha=-3 h+\sum_{i=1}^{10} e_{i}$. Then $\alpha^{2}=\alpha \cdot\left[K_{Y}\right]=-1$ so that $\alpha$ is a numerical exceptional curve. But there exists a nef and big divisor $H$ (for example $h$ ) such that $\alpha \cdot[H]<0$ so that $\alpha$ is not effective. Hence, $\alpha \cdot x \leq 0$ for all $x \in \bar{A}(Y)=\bar{A}_{\text {gen }}$ since $W^{\alpha}$ 
does not pass through the interior of $\overline{\mathcal{A}}_{\text {gen }}$. Note that $W^{\alpha}$ is never a face of $\overline{\mathscr{A}}_{\text {gen }}$. For $N=10, W^{-\alpha}$ is a face of $\overline{\mathscr{A}}_{\text {gen }}$, but this is no longer the case for $N \geq 11$. Thus, the condition $\alpha \cdot[H] \geq 0$ for some $H$ such that $H \cdot D>0$ is necessary for $\alpha$ to be effective.

More generally, let $f=3 h-\sum_{i=1}^{9} e_{i}$ and set $\alpha=k f+e_{10}$ (the case above corresponds to $k=-1$ ). As above, $\alpha$ is a numerical exceptional curve. For $k \leq-1$, $h \cdot \alpha<0$. Hence, $\alpha$ is not effective. For $k \geq 1, \alpha$ is effective but it is not the class of an exceptional curve: for all $x \in \bar{A}_{\text {gen }}, x \cdot f>0$, and $x \cdot e_{10} \geq 0$. Hence, $x \cdot \alpha>0$ for all $x \in \overline{\mathscr{A}}_{\text {gen }}$. Thus, $W^{\alpha}$ is not a face of $\bar{A}_{\text {gen }}$ and so $\alpha$ is not the class of an exceptional curve.

(ii) With $\alpha$ any of the classes as above, suppose that $N \geq 11$ and $k \neq 0$ and set $\beta=\alpha-e_{11}$. Then $\beta^{2}=-2$ and $\beta \cdot\left[K_{Y}\right]=0$. However,

$$
r_{\beta}\left(e_{11}\right)=e_{11}+\left(e_{11} \cdot \beta\right) \beta=\alpha .
$$

Since $W^{e_{11}}$ is a face of $\overline{\mathscr{A}}_{\text {gen }}$ and $W^{\alpha}$ is not a face of $\bar{A}_{\mathrm{gen}}, r_{\beta}\left(\overline{\mathscr{A}}_{\mathrm{gen}}\right) \neq \overline{\mathscr{A}}_{\mathrm{gen}}$. Hence, $\beta$ does not satisfy any of the equivalent conditions of Theorem 2.14 so that $\beta \notin R$.

Remark 2.20. In the situation of the example above, it is well known that if $D$ is irreducible, $N \leq 9$ (i.e., $D^{2} \geq 0$ ), and there are no -2-curves on $Y$, then every numerical exceptional curve is the class of an exceptional curve, so (i) above is best possible. A generalization is given in Proposition 3.3 below. We shall show in Proposition 3.5 that the example in (ii) is best possible as well.

The numerical exceptional curves given in Example 2.19(i) were known to $\mathrm{Du}$ Val. In fact, he showed that they are essentially the only numerical exceptional curves in case $Y$ is the blowup of $\mathbb{P}^{2}$ at ten points [Du Val 1937, pp. 46-47].

Proposition 2.21. Suppose that $(Y, D)$ is the blowup of $\mathbb{P}^{2}$ at ten points lying on an irreducible cubic, that $Y$ is generic in the sense that there are no-2-curves on $Y$, and that $\alpha$ is a numerical exceptional curve. Then there exists an exceptional curve $E$ on $Y$ and an integer $k$ such that $\alpha$ is the class of $k(D+E)+E$.

Proof. Suppose that $\alpha$ is a numerical exceptional curve on $Y$. Then, since $K_{Y}^{2}=-1$, $\lambda=\alpha+[D]=\alpha-\left[K_{Y}\right]$ satisfies $\lambda^{2}=\lambda \cdot \alpha=\lambda \cdot\left[K_{Y}\right]=0$. In particular, $\lambda \in \Lambda$. Conversely, given an isotropic vector $\lambda \in \Lambda$, if we set $\alpha=\lambda+\left[K_{Y}\right]$, then $\alpha$ is a numerical exceptional curve.

Any isotropic vector $\lambda \in \Lambda$ can be uniquely written as $n \lambda_{0}$, where $n \in \mathbb{Z}$ and $\lambda_{0}$ is primitive and lies in $\overline{\mathscr{C}^{+}}$. Note that $H^{2}(Y ; \mathbb{Z})=\mathbb{Z}\left[K_{Y}\right] \oplus \Lambda$ and that $\Lambda=U \oplus\left(-E_{8}\right)$ (both sums orthogonal). An easy exercise shows that, if $\operatorname{Aut}^{+}(\Lambda)$ is the group of integral isometries $A$ of $\Lambda$ such that $A\left(\mathscr{C}^{+} \cap \Lambda\right)=\mathscr{C}^{+} \cap \Lambda$, i.e., $A$ has real spinor norm equal to 1 , then every $A \in \operatorname{Aut}^{+}(\Lambda)$ extends uniquely to an integral isometry of $H^{2}(Y ; \mathbb{Z})$ fixing $\left[K_{Y}\right]$ and hence $[D]$ and moreover that $\operatorname{Aut}^{+}(\Lambda)$ acts transitively on the set of (nonzero) primitive isotropic vectors in $\overline{\mathscr{C}^{+}} \cap \Lambda$. Hence, there exists 
an $A \in \operatorname{Aut}^{+}(\Lambda)$ such that $A\left(\lambda_{0}\right)=f$ in the notation of Example 2.19. If we continue to let $A$ denote the extension of $A$ to an isometry of $H^{2}(Y ; \mathbb{Z})$, then $A(\alpha)=n f+\left[K_{Y}\right]=(n-1) f+e_{10}$ since $f=-\left[K_{Y}\right]+e_{10}$. It follows that $\alpha=(n-1) \lambda_{0}+A^{-1}\left(e_{10}\right)$. Using Proposition 3.5 below, $A^{-1}$ preserves the walls of the ample cone of $Y$, and thus, $A^{-1}\left(e_{10}\right)=e$ is the class of an exceptional curve $E$, and $\lambda_{0}=A^{-1}(f)=A^{-1}\left([D]+e_{10}\right)=[D]+E$. Hence, setting $k=n-1, \alpha$ is the class of $k(D+E)+E$ as claimed.

The proof above shows the following:

Corollary 2.22. Let $(Y, D)$ be the blowup of $\mathbb{P}^{2}$ at ten points lying on an irreducible cubic and such that there are no-2-curves on $Y$, let $\alpha$ be a numerical exceptional curve on $Y$, and let $\lambda=\alpha-\left[K_{Y}\right]$. Then

(i) $\alpha$ is effective if and only if $\lambda \in\left(\overline{\mathscr{C}^{+}}-\{0\}\right) \cap \Lambda$,

(ii) $\alpha$ is not effective if and only if $\lambda \in\left(-\overline{\mathscr{C}^{+}}\right) \cap \Lambda$, and

(iii) $\alpha$ is the class of an exceptional curve if and only if $\lambda$ is a primitive isotropic vector in $\overline{\mathscr{C}^{+}} \cap \Lambda$. Thus, there is a bijection from the set of exceptional curves on $Y$ to the set of primitive isotropic vectors in $\overline{\mathcal{C}^{+}} \cap \Lambda$.

Remark 2.23. In the above situation, let $\mathrm{W}$ be the group generated by the reflections in the classes $e_{1}-e_{2}, \ldots, e_{9}-e_{10}, h-e_{2}-e_{2}-e_{3}$, which are easily seen to be Looijenga roots. A classical argument (usually called Noether's inequality) shows that, if $\lambda_{0}$ is a primitive integral isotropic vector in $\Lambda$ lying in $\overline{\mathscr{C}^{+}}$, then there exists $w \in \mathrm{W}$ such that $w\left(\lambda_{0}\right)=f=3 h-\sum_{i=1}^{9} e_{i}$ in the notation of Example 2.19. Thus, $\mathrm{W}$ acts transitively on the set of such vectors. Using standard results about the affine Weyl group of $E_{8}$, it is then easy to see that $\mathrm{W}=\operatorname{Aut}^{+}(\Lambda)$. This was already noted in [Du Val 1937].

\section{Roots and the ample cone}

By Corollary 2.16, if $f: H^{2}(Y ; \mathbb{Z}) \rightarrow H^{2}(Y ; \mathbb{Z})$ is an integral isometry preserving the classes $\left[D_{i}\right]$ such that $f\left(\overline{\mathscr{A}}_{\text {gen }}\right)=\overline{\mathscr{A}}_{\text {gen }}$, then $f(R)=R$. In this section, we find criteria for when the converse holds.

Lemma 3.1. Let $f: H^{2}(Y ; \mathbb{Z}) \rightarrow H^{2}(Y ; \mathbb{Z})$ be an integral isometry preserving $\mathscr{C}^{+}$ and the classes $\left[D_{i}\right]$. If $f\left(\overline{\mathscr{A}}_{\mathrm{gen}}\right) \cap \overline{\mathscr{A}}_{\mathrm{gen}}$ contains an open set, then $f\left(\overline{\mathscr{A}}_{\mathrm{gen}}\right)=\overline{\mathscr{A}}_{\mathrm{gen}}$.

Proof. Choosing $x \in f\left(\overline{\mathscr{A}}_{\text {gen }}\right) \cap \overline{\mathcal{A}}_{\text {gen }}$ corresponding to an ample divisor, it is easy to see that $f\left(\overline{\mathscr{A}}_{\text {gen }}\right)$ and $\overline{\mathscr{A}}_{\text {gen }}$ have the same set of walls and hence are equal.

Next we deal with the case where one component of $D$ is a smooth rational curve of self-intersection -1 . 
Lemma 3.2. Suppose that $D$ is reducible and that $D_{r}^{2}=-1$. Let $(\bar{Y}, \bar{D})$ be the anticanonical pair obtained by contracting $D_{r}$. Then any isometry $f$ of $H^{2}(Y ; \mathbb{Z})$ preserving the classes $\left[D_{i}\right], 1 \leq i \leq r$, defines an isometry $\bar{f}$ of $H^{2}(\bar{Y} ; \mathbb{Z})$ preserving the classes $\left[\bar{D}_{i}\right], 1 \leq i \leq r-1$, and conversely. Moreover, $f$ preserves $\bar{A}_{\mathrm{gen}}(Y)$ if and only if $\bar{f}$ preserves $\bar{A}_{\mathrm{gen}}(\bar{Y})$, and $R_{Y}$ is naturally identified with the roots $R_{\bar{Y}}$ of $\bar{Y}$.

Proof. The first statement is clear. Identifying $H^{2}(\bar{Y}, \mathbb{Z})$ with $\left[D_{r}\right]^{\perp} \subseteq H^{2}(Y ; \mathbb{Z})$, it is clear that $\overline{\mathcal{A}}_{\text {gen }}(Y) \cap\left[D_{r}\right]^{\perp}=\overline{\mathscr{A}}_{\text {gen }}(\bar{Y})$. Hence, if $f$ preserves $\overline{\mathscr{A}}_{\text {gen }}(Y)$, then $\bar{f}$ preserves $\bar{A}_{\text {gen }}(\bar{Y})$. Since a divisor $\bar{H}$ on $\bar{Y}$ is ample if and only if $N \bar{H}-D_{r}$ is ample for all $N \gg 0$, it follows that, if $\bar{f}$ preserves $\bar{A}_{\text {gen }}(\bar{Y})$, then $f\left(\overline{\mathscr{A}}_{\text {gen }}(Y)\right) \cap \overline{\mathcal{A}}_{\text {gen }}(Y)$ contains an open set, and hence, $f\left(\overline{\mathscr{A}}_{\text {gen }}(Y)\right)=\overline{\mathscr{A}}_{\text {gen }}(Y)$ by Lemma 3.1. It follows from this and from Theorem 2.14 that $R_{Y}$ is naturally identified with $R_{\bar{Y}}$ (or directly from the definition by noting that there is a bijection from the set of deformations of $(Y, D)$ to those of $(\bar{Y}, \bar{D}))$.

Henceforth, then, we shall always assume if need be that no component of $D$ is a smooth rational curve of self-intersection -1 .

We turn to the straightforward case where $(Y, D)$ is not negative definite.

Proposition 3.3. Suppose that $(Y, D)$ and $\left(Y^{\prime}, D^{\prime}\right)$ are two anticanonical pairs with $r(D)=r\left(D^{\prime}\right)$ and neither pair is negative definite. If $f: H^{2}(Y ; \mathbb{Z}) \rightarrow H^{2}\left(Y^{\prime} ; \mathbb{Z}\right)$ is an integral isometry with $f\left(\left[D_{i}\right]\right)=\left[D_{i}^{\prime}\right]$ for all $i$, then $f\left(\overline{\mathscr{A}}_{\mathrm{gen}}(Y)\right)=\overline{\mathscr{A}}_{\mathrm{gen}}\left(Y^{\prime}\right)$ and hence $f\left(R_{Y}\right)=R_{Y^{\prime}}$. Moreover,

$$
R_{Y}=\left\{\beta \in \Lambda(Y, D): \beta^{2}=-2\right\} .
$$

Proof. By Lemma 3.2, we may assume that no $D_{i}$ has self-intersection -1 . The statement that the cycle is not negative definite is then equivalent to the statement that either $D_{j}^{2} \geq 0$ for some $j$ or $D_{i}^{2}=-2$ for all $i$ and $r \geq 2$. In the first case, $D_{j}$ is nef and $D_{j} \cdot D>0$. Hence, if $\alpha$ is a numerical exceptional curve such that $\alpha \cdot\left[D_{j}\right] \geq 0$, then $\alpha$ is effective by Lemma 2.4. Thus, $\bar{A}_{\text {gen }}(Y)$ is the set of all $x \in \mathscr{C}_{D}^{+}(Y)$ such that $x \cdot \alpha \geq 0$ for all numerical exceptional curves $\alpha$ such that $\alpha \cdot\left[D_{j}\right] \geq 0$. Since $f(\alpha)^{2}=\alpha^{2}, f\left(\left[D_{j}\right]\right)=\left[D_{j}^{\prime}\right]$, and $f(\alpha) \cdot\left[K_{Y^{\prime}}\right]=\alpha \cdot\left[K_{Y}\right]$, it follows that $f\left(\overline{\mathscr{A}}_{\text {gen }}(Y)\right)=\overline{\mathscr{A}}_{\text {gen }}\left(Y^{\prime}\right)$. Applying this to reflection in a class $\beta$ of square -2 in $\Lambda(Y, D)$ then implies that $\beta \in R_{Y}$.

The case where $D_{i}^{2}=-2$ for every $i$ is similar, using the nef divisor $D=\sum_{i} D_{i}$ with $D^{2}=0$. If $\alpha$ is a numerical exceptional curve, then $\alpha$ is effective since $\left(-\alpha+\left[K_{Y}\right]\right) \cdot[D]=\alpha \cdot\left[K_{Y}\right]=-1$. The rest of the argument proceeds as before.

Remark 3.4. If $D$ is irreducible and not negative definite (i.e., $D^{2} \geq 0$ ) and there are no -2-curves on $Y$, then, as is well known and noted in Remark 2.20, every numerical exceptional curve is the class of an exceptional curve. However, if $D$ is reducible but not negative definite, then, even if there are no-2-curves on $Y$, there 
may well exist numerical exceptional curves that are not effective and effective numerical exceptional curves that are not the class of an exceptional curve.

From now on, we assume that $D$ is negative definite. The case $K_{Y}^{2}=-1$ can also be handled by straightforward methods as noted in [Looijenga 1981]. (See also [Friedman and Morgan 1988, II (2.7)(c)] in case $D$ is irreducible.)

Proposition 3.5. Let $(Y, D)$ and $\left(Y^{\prime}, D^{\prime}\right)$ be two negative definite anticanonical pairs with $r(D)=r\left(D^{\prime}\right)$ and $K_{Y}^{2}=K_{Y^{\prime}}^{2}=-1$. Let $f: H^{2}(Y ; \mathbb{Z}) \rightarrow H^{2}\left(Y^{\prime} ; \mathbb{Z}\right)$ be an isometry such that $f\left(\left[D_{i}\right]\right)=\left[D_{i}^{\prime}\right]$ for all $i$ and $f\left(\mathscr{C}^{+}(Y)\right)=\mathscr{C}^{+}\left(Y^{\prime}\right)$. Then $f\left(\bar{A}_{\text {gen }}(Y)\right)=\bar{A}_{\text {gen }}\left(Y^{\prime}\right)$. Moreover,

$$
R_{Y}=\left\{\beta \in \Lambda(Y, D): \beta^{2}=-2\right\} .
$$

Hence, $f\left(R_{Y}\right)=R_{Y^{\prime}}$.

Proof. Since $(Y, D)$ is negative definite, no component of $D$ is a smooth rational curve of self-intersection -1 . Fix a nef and big divisor $H$ such that $H \cdot D_{i}=0$ for all $i$ and $H \cdot G>0$ for every irreducible curve $G \neq D_{i}$. If $\alpha$ is a numerical exceptional curve, $\left(\alpha-\left[K_{Y}\right]\right)^{2}=(\alpha+[D])^{2}=0$. By Lemma 2.6, $\alpha$ is effective if and only if $[H] \cdot \alpha>0$ if and only if $[H] \cdot(\alpha+[D])>0$. By the light cone lemma [Friedman and Morgan 1988, p. 320], this last condition is equivalent to $\alpha+[D] \in \overline{\mathscr{C}^{+}}-\{0\}$. Since this condition is clearly preserved by an isometry $f$ as in the statement of the proposition, we see that $f\left(\overline{\mathscr{A}}_{\text {gen }}(Y)\right)=\overline{\mathscr{A}}_{\text {gen }}\left(Y^{\prime}\right)$. The final statement then follows as in the proof of Proposition 3.3.

Remark 3.6. The hypothesis $K_{Y}^{2}=-1$ implies that $r(D) \leq 10$, so there are only finitely many examples of the above type. For $r(D)=10$, there is essentially just one combinatorial possibility for $(Y, D)$ neglecting the orientation [Friedman and Miranda 1983, (4.7)], where it is easy to check that this is the only possibility. For $r(D)=9$, however, there are two different possibilities for the combinatorial type of $(Y, D)$ (again ignoring the orientation). Begin with an anticanonical pair $(\bar{Y}, \bar{D})$, where $\bar{Y}$ is a rational elliptic surface and $\bar{D}=\bar{D}_{0}+\cdots+\bar{D}_{8}$ is a fiber of type $\tilde{A}_{8}$ (or $I_{9}$ in Kodaira's notation). There is a unique such rational elliptic surface $\bar{Y}$, and its Mordell-Weil group has order 3 (see, for example, [Miranda and Persson 1986]). In particular, possibly after relabeling the components, there is an exceptional curve meeting $\bar{D}_{i}$ if and only if $i=0,3,6$. It is easy to see that blowing up a point on a component $\bar{D}_{i}$ meeting an exceptional curve leads to a different combinatorial possibility for an anticanonical pair $(Y, D)$ with $K_{Y}^{2}=-1$ and $r(D)=9$ than blowing up a point on a component $\bar{D}_{i}$ that does not meet an exceptional curve.

We turn now to the case where $(Y, D)$ is negative definite but with no assumption on $K_{Y}^{2}$. 
Definition 3.7. A point $x \in \mathscr{C}^{+} \cap \Lambda$ is $R$-distinguished if there exists a codimension-1 negative definite subspace $V$ of $\Lambda \otimes \mathbb{R}$ spanned by elements of $R$ such that $x \in V^{\perp}$. Note that the definition only depends on the deformation type of the pair $(Y, D)$.

Remark 3.8. Clearly, if $V$ is a codimension-1 negative definite subspace of $\Lambda \otimes \mathbb{R}$ spanned by elements of $R$, then $V$ is defined over $\mathbb{Q}$ and $V^{\perp} \cap(\Lambda \otimes \mathbb{R})$ is a onedimensional subspace of $H^{2}(Y ; \mathbb{R})$ defined over $\mathbb{Q}$ and spanned by an $h \in H^{2}(Y ; \mathbb{Z})$ with $h^{2}>0, h \cdot\left[D_{i}\right]=0$, and $h \cdot \beta=0$ for all $\beta \in R \cap V$. Hence, if $h \in \mathscr{C}^{+} \cap \Lambda$, then $h$ is $R$-distinguished.

Also, if the rank of $\Lambda$ is one, then $\{0\}$ is a codimension- 1 negative definite subspace of $\Lambda \otimes \mathbb{R}$, and hence, every point of $\mathscr{C}^{+} \cap \Lambda$ is $R$-distinguished.

However, as we shall see, there exist deformation types $(Y, D)$ with no $R$ distinguished points.

The following is also clear:

Lemma 3.9. Let $(Y, D)$ and $\left(Y^{\prime}, D^{\prime}\right)$ be two anticanonical pairs with $r(D)=r\left(D^{\prime}\right)$, and let $f: H^{2}(Y ; \mathbb{Z}) \rightarrow H^{2}\left(Y^{\prime} ; \mathbb{Z}\right)$ be an isometry such that $f\left(\left[D_{i}\right]\right)=\left[D_{i}^{\prime}\right]$ for all $i$, $f\left(\mathscr{C}^{+}(Y)\right)=\mathscr{C}^{+}\left(Y^{\prime}\right)$, and $f\left(R_{Y}\right)=R_{Y^{\prime}}$. Then, if $x$ is an $R_{Y}$-distinguished point of $\mathscr{C}^{+}(Y) \cap \Lambda(Y, D), f(x)$ is an $R_{Y^{\prime}}$-distinguished point of $\mathscr{C}^{+}\left(Y^{\prime}\right) \cap \Lambda\left(Y^{\prime}, D^{\prime}\right)$.

Our goal now is to prove this:

Theorem 3.10. Suppose that $(Y, D)$ and $\left(Y^{\prime}, D^{\prime}\right)$ are two anticanonical pairs such that $r(D)=r\left(D^{\prime}\right)$. Let $f: H^{2}(Y ; \mathbb{Z}) \rightarrow H^{2}\left(Y^{\prime} ; \mathbb{Z}\right)$ be an isometry such that $f\left(\left[D_{i}\right]\right)=\left[D_{i}^{\prime}\right]$ for all $i, f\left(\mathscr{C}^{+}(Y)\right)=\mathscr{C}^{+}\left(Y^{\prime}\right)$, and $f\left(R_{Y}\right)=R_{Y^{\prime}}$. If there exists an $R$-distinguished point of $\mathscr{C}^{+} \cap \Lambda$, then $f\left(\bar{A}_{\text {gen }}(Y)\right)=\bar{A}_{\text {gen }}\left(Y^{\prime}\right)$.

We begin by showing:

Proposition 3.11. Let $x$ be an R-distinguished point of $\mathscr{C}^{+} \cap \Lambda$. Then $x \in \overline{\mathcal{A}}_{\text {gen }}$. Moreover, if $\alpha$ is a numerical exceptional curve and $\alpha$ is not in the span of the $\left[D_{j}\right]$, then $\alpha$ is effective if and only if $\alpha \cdot x \geq 0$.

Proof. It is enough by Lemma 2.9 to check this on some (global) deformation of $(Y, D)$ with trivial monodromy. By Theorem 1.3, we can assume that

$$
\operatorname{Ker} \varphi_{Y}=V \cap \Lambda,
$$

where $V$ is as in the definition of $R$-distinguished. In particular, if $C \in \Delta_{Y}$, i.e., $C$ is a-2-curve on $Y$, then $[C] \in V$. It follows from Theorem 2.14(i) that every $\beta \in V \cap R$ is a sum of elements of $\Delta_{Y}$ so that $\Delta_{Y}$ spans $V$ over $\mathbb{Q}$. Thus, there exist -2-curves $C_{1}, \ldots, C_{k}$ such that $V$ is spanned by the classes $\left[C_{i}\right]$, and the intersection matrix $\left(C_{i} \cdot C_{j}\right)$ is negative definite. The classes $\left[C_{1}\right], \ldots,\left[C_{k}\right],\left[D_{1}\right], \ldots,\left[D_{r}\right]$ span a negative definite sublattice of $H^{2}(Y ; \mathbb{Z})$. By Proposition 1.5, there exists a nef and big divisor $H$ such that $H$ is perpendicular to the curves $C_{1}, \ldots, C_{k}, D_{1}, \ldots, D_{r}$. 
Clearly, then $[H] \in \bar{A}(Y) \subseteq \overline{\mathcal{A}}_{\text {gen }}$ and $[H]=t x$ for some $t \in \mathbb{R}^{+}$. Hence, $x \in \bar{A}_{\text {gen }}$ as well. Note that $[H]^{\perp}$ is spanned over $\mathbb{Q}$ by $\left[C_{1}\right], \ldots,\left[C_{k}\right],\left[D_{1}\right], \ldots,\left[D_{r}\right]$.

Since $x \in \bar{A}(Y)$, if $\alpha$ is effective, $x \cdot \alpha \geq 0$. Conversely, suppose that $\alpha$ is a numerical exceptional curve with $x \cdot \alpha \geq 0$ and that $\alpha$ is not effective. Then $-\alpha+\left[K_{Y}\right]=[G]$, where $G$ is effective, and $H \cdot\left(-\alpha+\left[K_{Y}\right]\right)=-\alpha \cdot[H] \leq 0$. Hence, $\left(-\alpha+\left[K_{Y}\right]\right) \cdot[H]=0$.

Claim 3.12. We have $-\alpha+\left[K_{Y}\right]=\sum_{i} a_{i}\left[C_{i}\right]+\sum_{j} b_{j}\left[D_{j}\right]$, where $a_{i}, b_{j} \in \mathbb{Z}$.

Proof. In any case, since $-\alpha+\left[K_{Y}\right]$ is perpendicular to $[H]$, there must exist $a_{i}, b_{j} \in \mathbb{Q}$ such that $-\alpha+\left[K_{Y}\right]=\sum_{i} a_{i}\left[C_{i}\right]+\sum_{j} b_{j}\left[D_{j}\right]$. There exist $n_{i}, m_{j} \in \mathbb{Z}$ such that $-\alpha+\left[K_{Y}\right]=[G]=\sum_{i} n_{i}\left[C_{i}\right]+\sum_{j} m_{j}\left[D_{j}\right]+[F]$, where $F$ is an effective curve not containing $C_{i}$ or $D_{j}$ in its support for any $i, j$. By Lemma 1.4(ii), $F=0$, $a_{i}=n_{i}$, and $b_{j}=m_{j}$ for all $i, j$. Hence, $a_{i}, b_{j} \in \mathbb{Z}$.

Because $-\alpha+\left[K_{Y}\right]$ is an integral linear combination of the $\left[C_{i}\right]$ and $\left[D_{j}\right]$, the same holds for $\alpha$. Then $\alpha=\sum_{i} c_{i}\left[C_{i}\right]+\sum_{j} d_{j}\left[D_{j}\right]$ with $c_{i}, d_{j} \in \mathbb{Z}$. However, $\alpha^{2}=-1=\left(\sum_{i} c_{i} C_{i}\right)^{2}+\left(\sum_{j} d_{j} D_{j}\right)^{2}$. Both terms are nonpositive, and so $\left(\sum_{i} c_{i} C_{i}\right)^{2} \geq-1$. But if $\sum_{i} c_{i} C_{i} \neq 0$, then $\left(\sum_{i} c_{i} C_{i}\right)^{2} \leq-2$. Thus, $\sum_{i} c_{i} C_{i}=0$ and $\alpha$ lies in the span of the $\left[D_{j}\right]$. Conversely, if $\alpha$ is not in the span of the $\left[D_{j}\right]$ and $\alpha \cdot x \geq 0$, then $\alpha$ is the class of an effective curve.

Proof of Theorem 3.10. It follows from Proposition 3.11 that, if $x \in \mathscr{C}^{+}(Y) \cap \Lambda(Y, D)$ is $R_{Y}$-distinguished, then $\bar{A}_{\text {gen }}(Y)$ is the set of all $y \in \mathscr{C}_{D}^{+}(Y)$ such that $\alpha \cdot y \geq 0$ for all $\alpha$ a numerical exceptional curve on $Y$, not in the span of the $\left[D_{i}\right]$, such that $\alpha \cdot x \geq 0$. Let $f$ be an isometry satisfying the conditions of the theorem. Then $f(x)$ is $R_{Y^{\prime}}$-distinguished, and $f\left(\overline{\mathscr{A}}_{\text {gen }}(Y)\right)$ is clearly the set of all $y \in \mathscr{C}_{D^{\prime}}^{+}\left(Y^{\prime}\right)$ such that $\alpha \cdot y \geq 0$ for all $\alpha$ a numerical exceptional curve on $Y^{\prime}$, not in the span of the $\left[D_{i}^{\prime}\right]$, such that $\alpha \cdot f(x) \geq 0$. Again by Proposition 3.11, this set is exactly $\bar{A}_{\text {gen }}\left(Y^{\prime}\right)$.

Theorem 3.10 covers all of the cases in [Looijenga 1981] except for the case of five components: by inspection of the root diagrams on [Looijenga 1981, pp. 275277], the complement of any trivalent vertex spans a negative definite codimension-1 subspace except in the case of five components. To give a direct argument along the above lines that also handles this case (and all of the other cases in [Looijenga 1981]), we recall the basic setup there: there exists a subset $B=\left\{\beta_{1}, \ldots, \beta_{n}\right\} \subseteq R$ such that $B$ is a basis for $\Lambda \otimes \mathbb{R}$, and there exist $n_{i} \in \mathbb{Z}^{+}$such that $\left(\sum_{i} n_{i} \beta_{i}\right) \cdot \beta_{j}>0$ for all $j$ (compare also [Looijenga 1980, (1.18)]). In particular, note that the intersection matrix $\left(\beta_{i} \cdot \beta_{j}\right)$ is nonsingular. Finally, by the classification of [Looijenga 1981, Theorem (1.1)], there exists a deformation of $(Y, D)$ for which $\beta_{i}=\left[C_{i}\right]$ is the class of a -2-curve for all $i$. (With some care, this explicit argument could be avoided by appealing to the surjectivity of the period map and Theorem 2.14(i).) 
Theorem 3.13. Let $(Y, D)$ and $\left(Y^{\prime}, D^{\prime}\right)$ be two anticanonical pairs satisfying the hypotheses of the preceding paragraph, both negative definite, with $r(D)=r\left(D^{\prime}\right)$, and let $f: H^{2}(Y ; \mathbb{Z}) \rightarrow H^{2}\left(Y^{\prime} ; \mathbb{Z}\right)$ be an isometry such that $f\left(\left[D_{i}\right]\right)=\left[D_{i}^{\prime}\right]$ for all $i, f\left(\mathscr{C}^{+}(Y)\right)=\mathscr{C}^{+}\left(Y^{\prime}\right)$, and $f\left(R_{Y}\right)=R_{Y^{\prime}}$. Then $f\left(\overline{\mathcal{A}}_{\mathrm{gen}}(Y)\right)=\overline{\mathscr{A}}_{\mathrm{gen}}\left(Y^{\prime}\right)$.

Sketch of the proof. With notation as in the paragraph preceding the statement of the theorem, let $h=\sum_{i} n_{i} \beta_{i}$ have the property that $h \cdot \beta_{i}>0$. By the arguments used in the proof of Theorem 3.10, it is enough to show that $h \in \overline{\mathcal{A}}_{\text {gen }}$ and that, if $\alpha$ is a numerical exceptional curve and $\alpha$ is not in the span of the $\left[D_{j}\right]$, then $\alpha$ is effective if and only if $\alpha \cdot h \geq 0$. Also, it is enough to prove this for some deformation of $(Y, D)$, so we can assume $\beta_{i}=\left[C_{i}\right]$ is the class of a -2-curve for all $i$ and hence that $h$ is the class of $H=\sum_{i} n_{i} C_{i}$. By construction, $H \cdot C_{j}>0$ for every $j$. Hence, $H$ is nef and big. By Lemma 2.6, it is enough to show that, if $G$ is an irreducible curve not equal to $D_{i}$ for any $i$, then $H \cdot G>0$. Since $H$ is nef, it suffices to rule out the case $H \cdot G=0$, in which case $G^{2}<0$. As $G \neq D_{j}$ for any $j$, then $G$ is either a -2-curve or an exceptional curve. The case where $G$ is a -2-curve is impossible since then $G$ is orthogonal to the span of the $\left[C_{i}\right]$, but the $\left[C_{i}\right]$ span $\Lambda$ over $\mathbb{Q}$ and the intersection form is nondegenerate. So $G=E$ is an exceptional curve disjoint from the $C_{i}$. If $(\bar{Y}, \bar{D})$ is the anticanonical pair obtained by contracting $E$, then the $\left[C_{i}\right]$ define classes in $\bar{\Lambda}=\Lambda(\bar{Y}, \bar{D})$. Since the intersection form $\left(C_{i} \cdot C_{j}\right)$ is nondegenerate, the rank of $\bar{\Lambda}$ is at least that of $\Lambda$. It is easy to check that the classes of $\bar{D}_{1}, \ldots, \bar{D}_{r}$ are linearly independent: if say $E$ meets $D_{1}$, then the intersection matrix of $\bar{D}_{2}, \ldots, \bar{D}_{r}$ is still negative definite and then Lemma 1.4(ii) (with $F=\bar{D}_{1}$ and $G_{1}, \ldots, G_{n}=\bar{D}_{2}, \ldots, \bar{D}_{r}$ ) shows that the classes of $\bar{D}_{1}, \ldots, \bar{D}_{r}$ are linearly independent. Hence, the rank of $H^{2}(\bar{Y} ; \mathbb{Z})$ is greater than or equal to the rank of $H^{2}(Y ; \mathbb{Z})$, which contradicts the fact that $\bar{Y}$ is obtained from $Y$ by contracting an exceptional curve.

\section{Some examples}

Example 4.1. We provide a series of examples that satisfy the hypotheses of Theorem 3.10, where the number of components and the multiplicities are arbitrarily large. Let $(\bar{Y}, \bar{D})$ be the anticanonical pair obtained by making $k+6$ infinitely near blowups starting with the double point of a nodal cubic. Thus, $\bar{D}=\bar{D}_{0}+\cdots+\bar{D}_{k+6}$, where $\bar{D}_{0}^{2}=-k, \bar{D}_{i}^{2}=-2,1 \leq i \leq k+5$, and $\bar{D}_{k+6}^{2}=-1$. Now blow up $N \geq 1$ points $p_{1}, \ldots, p_{N}$ on $\bar{D}_{k+6}$, and let $(Y, D)$ be the resulting anticanonical pair. Note that $(Y, D)$ is negative definite as long as $k \geq 3$ or $k=2$ and $N \geq 2$. Clearly $r(D)=k+7$ and $K_{Y}^{2}=3-k-N$. It follows that $\Lambda=\Lambda(Y, D)$ has rank $N$. If $E_{1}, \ldots, E_{N}$ are the exceptional curves corresponding to $p_{1}, \ldots, p_{N}$, then the classes $\left[E_{i}\right]-\left[E_{i+1}\right]$ span a negative definite root lattice of type $A_{N-1}$ in $\Lambda$. By making all of the blowups 
infinitely near to the first point, we see that all of the classes $\left[E_{i}\right]-\left[E_{i+1}\right]$ lie in $R$. Hence, $(Y, D)$ satisfies the hypotheses of Theorem 3.10.

Next we turn to examples where the rank of $\Lambda$ is small. The case where the rank of $\Lambda$ is 1 is covered by Theorem 3.10 as well as the case where the rank of $\Lambda$ is 2 and $R \neq \varnothing$. Note that, conjecturally at least, the case where $R \neq \varnothing$ should be related to the question of whether the dual cusp singularity deforms to an ordinary double point. It is easy to construct examples where the rank of $\Lambda$ is 2 and with $R \neq \varnothing$ : begin with an anticanonical pair $(\hat{Y}, \hat{D})$ where the rank of $\Lambda(\hat{Y}, \hat{D})$ is 1 , locate a component $\hat{D}_{i}$ such that there exists an exceptional curve $E$ on $\hat{Y}$ with $E \cdot \hat{D}_{i}=1$, and blow up a point of $\hat{D}_{i}$ to obtain a new anticanonical pair $(Y, D)$ together with exceptional curves $E$ and $E^{\prime}$ (where we continue to let $E$ denote the pullback to $Y$ and $E^{\prime}$ the new exceptional curve) such that $[E]-\left[E^{\prime}\right] \in R$. So our interest is in finding examples where $R=\varnothing$.

Remark 4.2. In case the rank of $\Lambda$ is 2 and $R \neq \varnothing$, it is easy to see that either $\left(\overline{\mathscr{A}}_{\text {gen }} \cap \Lambda\right) / \mathbb{R}^{+}$is a closed (compact) interval or $\overline{\mathscr{A}}_{\text {gen }} \cap \Lambda=\mathscr{C}^{+} \cap \Lambda$ (and in fact both cases arise). In either case, there is at most one wall $W^{\beta}$ with $\beta \in R$ passing through the interior of $\overline{\mathscr{A}}_{\text {gen }} \cap \Lambda$, and hence, either $R=\varnothing$ or $R=\{ \pm \beta\}$.

Example 4.3. We give an example where the rank of $\Lambda$ is 2 and there are no $\beta \in \Lambda$ such that $\beta^{2}=-2$ (in particular, $R=\varnothing$; hence, the condition $f(R)=R$ is automatic for every isometry $f$ ) and of an isometry $f$ that preserves $\mathscr{C}^{+}$and the classes $\left[D_{i}\right]$ but not the generic ample cone. Let $(\bar{Y}, \bar{D})$ be the anticanonical pair obtained by making nine infinitely near blowups starting with the double point of a nodal cubic. Thus, $\bar{D}=\bar{D}_{0}+\cdots+\bar{D}_{9}$, where $\bar{D}_{0}=3 H-2 E_{1}-\sum_{i=2}^{9} E_{i}$, $\bar{D}_{i}=E_{i}-E_{i+1}, 1 \leq i \leq 8$, and $\bar{D}_{9}=E_{9}$. Make two more blowups, one at a point $p_{10}$ on $\bar{D}_{9}$ and one at a point $p_{11}$ on $\bar{D}_{4}$. This yields an anticanonical pair $(Y, D)$ with $D_{0}=3 H-2 E_{1}-\sum_{i=2}^{9} E_{i}, D_{i}=E_{i}-E_{i+1}, i>0$ and $i \neq 4$, and $D_{4}=E_{4}-E_{5}-E_{11}$. Thus,

$$
\left(-d_{0}, \ldots,-d_{9}\right)=(3,2,2,2,3,2,2,2,2,2),
$$

i.e., $D$ is of type $\left(\begin{array}{ll}3 & 3 \\ 3 & 5\end{array}\right)$, with dual cycle $\left(\begin{array}{ll}6 & 8 \\ 0 & 0\end{array}\right)$ in the notation of [Friedman and Miranda 1983]. Set

$G_{1}=5 H-2 \sum_{i=1}^{4} E_{i}-\sum_{i=5}^{10} E_{i}-E_{11} \quad$ and $\quad G_{2}=10 H-5 \sum_{i=1}^{4} E_{i}-\sum_{i=5}^{10} E_{i}-4 E_{11}$.

It is straightforward to check that $\left(G_{i} \cdot D_{j}\right)=0$ for $i=1,2$ and $0 \leq j \leq 9$. Hence, $G_{1}, G_{2} \in \Lambda$. Also,

$$
G_{1}^{2}=2, \quad G_{2}^{2}=-22, \quad G_{1} \cdot G_{2}=0
$$


The corresponding quadratic form

$$
q(n, m)=\left(n G_{1}+m G_{2}\right)^{2}=2 n^{2}-22 m^{2}
$$

has discriminant $-44=-2^{2} \cdot 11$. Note that this is consistent with the fact that the discriminant of the dual cycle is

$$
\operatorname{det}\left(\begin{array}{rr}
-6 & 2 \\
2 & -8
\end{array}\right)=44
$$

It is easy to see that $G_{1}$ and $G_{2}$ are linearly independent mod 2 and hence span a primitive lattice, which must therefore equal $\Lambda$.

First, we claim that there is no element of $\Lambda$ of square -2 . This is equivalent to the statement that there is no solution in integers to the equation $n^{2}-11 m^{2}=-1$, i.e., that the fundamental unit in $\mathbb{Z}[\sqrt{11}]$ has norm 1 . But clearly if there were an integral solution to $n^{2}-11 m^{2}=-1$, then since $-11 \equiv 1 \bmod 4$, we could write -1 as a sum of two squares mod 4 , which is impossible. In fact, the fundamental unit in $\mathbb{Z}[\sqrt{11}]$ is $10+3 \sqrt{11}$. Thus, if $R$ is the set of roots for $(Y, D)$, then $R=\varnothing$. In particular, any isometry $f$ trivially satisfies $f(R)=R$.

Finally, we claim there is an isometry $f$ of $H^{2}(Y ; \mathbb{Z})$ such that $f\left(\left[D_{i}\right]\right)=\left[D_{i}\right]$ for all $i$ and $f\left(\mathscr{C}^{+}\right)=\mathscr{C}^{+}$but such that $f$ does not preserve the generic ample cone. Note the unit group $U$ of $\mathbb{Z}[\sqrt{11}]$ acts as a group of isometries on $\Lambda$ and hence acts as a group of isometries (with $\mathbb{Q}$-coefficients) of the lattice

$$
H^{2}(Y ; \mathbb{Q})=(\Lambda \otimes \mathbb{Q}) \oplus \bigoplus_{i} \mathbb{Q}\left[D_{i}\right]
$$

fixing the classes $\left[D_{i}\right]$. Also, any isometry of $\Lambda$ that is trivial on the discriminant group $\Lambda^{\vee} / \Lambda$ extends to an integral isometry of $H^{2}(Y ; \mathbb{Z})$ fixing the $\left[D_{i}\right]$. Concretely, the discriminant form $\Lambda^{\vee} / \Lambda \cong \mathbb{Z} / 2 \mathbb{Z} \oplus \mathbb{Z} / 22 \mathbb{Z}$. If $\mu=10+3 \sqrt{11}$, then it is easy to check that the automorphism of $\Lambda$ corresponding to $\mu^{2}=199+60 \sqrt{11}$ acts trivially on $\Lambda^{\vee} / \Lambda$ and hence defines an isometry $f$ of $H^{2}(Y ; \mathbb{Z})$ fixing the $\left[D_{i}\right]$. Then $f$ acts freely on $\left(\mathscr{C}^{+} \cap \Lambda\right) / \mathbb{R}^{+}$, which is just a copy of $\mathbb{R}$ (and $f$ acts on it via translation). But the intersection of the generic ample cone with $\Lambda$ has the nontrivial wall $W^{E_{11}}$ so that the intersection cannot be all of $\mathscr{C}^{+} \cap \Lambda$. It follows that $f^{ \pm 1}$ does not preserve the generic ample cone. Explicitly, let $(\hat{Y}, \hat{D})$ be the surface obtained by contracting $E_{11}$ and let $\hat{G}_{1}=4 G_{1}-G_{2}=10 H-3 \sum_{i=1}^{10} E_{i}$ be the pullback of the positive generator of $\Lambda(\hat{Y}, \hat{D})$. Thus, $\hat{G}_{1}$ is nef and big so that $\hat{G}_{1} \in \overline{\mathcal{A}}_{\text {gen }}$. Clearly $\hat{G}_{1} \in W^{E_{11}}$. If $A=\left(\begin{array}{cc}a & 11 b \\ b & a\end{array}\right)$ is the isometry of $\Lambda$ corresponding to multiplication by the unit $a+b \sqrt{11}$, then $A\left(G_{1}\right)=a G_{1}+b G_{2}, A\left(G_{2}\right)=11 b G_{1}+a G_{2}$, and $A\left(\hat{G}_{1}\right)=(4 a-11 b) G_{1}+(4 b-a) G_{2}$. Thus,

$$
E_{11} \cdot A\left(\hat{G}_{1}\right)=(4 a-11 b)+4(4 b-a)=5 b .
$$


Hence, $E_{11} \cdot A\left(\hat{G}_{1}\right)<0$ if $b<0$. Taking $f^{-1}$, which corresponds to $199-60 \sqrt{11}$, we see that $f^{-1}\left(\hat{G}_{1}\right) \notin \bar{A}_{\text {gen }}$.

Example 4.4. In this example, the rank of $\Lambda$ is 2 and $R=\varnothing$, but there exist infinitely many $\beta \in \Lambda$ such that $\beta^{2}=-2$. The condition $f(R)=R$ is again automatic for every isometry $f$, and reflection about every $\beta \in \Lambda$ with $\beta^{2}=-2$ is an isometry that preserves $\mathscr{C}^{+}$and the classes $\left[D_{i}\right]$ but not the generic ample cone.

As in the previous example, let $(\bar{Y}, \bar{D})$ be the anticanonical pair obtained by making nine infinitely near blowups starting with the double point of a nodal cubic. Thus, $\bar{D}=\bar{D}_{0}+\cdots+\bar{D}_{9}$, where $\bar{D}_{0}=3 H-2 E_{1}-\sum_{i=2}^{9} E_{i}, \bar{D}_{i}=E_{i}-E_{i+1}$, $1 \leq i \leq 8$, and $\bar{D}_{9}=E_{9}$. Make two more blowups, one at a point $p_{10}$ on $\bar{D}_{9}$ and one at a point $p_{11}$ on $\bar{D}_{0}$. This yields an anticanonical pair $(Y, D)$ with $D_{0}=3 H-2 E_{1}-\sum_{i=2}^{9} E_{i}-E_{11}$ and $D_{i}=E_{i}-E_{i+1}, 1 \leq i \leq 9$. Thus,

$$
\left(-d_{0}, \ldots,-d_{9}\right)=(4,2,2,2,2,2,2,2,2,2),
$$

i.e., $D$ is of type $\left(\begin{array}{l}4 \\ 9\end{array}\right)$, with dual cycle $\left(\begin{array}{c}12 \\ 1\end{array}\right)$ in the notation of [Friedman and Miranda 1983]. Set

$$
G_{1}=10 H-3 \sum_{i=1}^{10} E_{i} \quad \text { and } \quad G_{2}=3 H-\sum_{i=1}^{10} E_{i}+E_{11} .
$$

It is straightforward to check that $\left(G_{i} \cdot D_{j}\right)=0$ for $i=1,2$ and $0 \leq j \leq 9$. Hence, $G_{1}, G_{2} \in \Lambda$. Also,

$$
G_{1}^{2}=10, \quad G_{2}^{2}=-2, \quad \text { and } \quad G_{1} \cdot G_{2}=0 .
$$

The corresponding quadratic form

$$
q(n, m)=\left(n G_{1}+m G_{2}\right)^{2}=10 n^{2}-2 m^{2}
$$

has discriminant $-20=-2^{2} \cdot 5$. Note that this is consistent with the fact that the discriminant of the dual cycle is

$$
\operatorname{det}\left(\begin{array}{cr}
-12 & 2 \\
2 & -2
\end{array}\right)=20
$$

It is easy to see that $G_{1}$ and $G_{2}$ are linearly independent mod 2 and hence span a primitive lattice, which must therefore equal $\Lambda$.

To give a partial description of $\overline{\mathcal{A}}_{\text {gen }} \cap \Lambda$, note that (as for $\hat{G}_{1}$ in the previous example) $G_{1}$ is the pullback to $Y$ of a positive generator for $\Lambda(\hat{Y}, \hat{D})$, where $\hat{Y}$ denotes the surface obtained by contracting $E_{11}$. Thus, $G_{1}$ is nef and big so that $G_{1} \in \overline{\mathcal{A}}_{\text {gen }}$ and also $G_{1} \in W^{E_{11}}$. Hence,

$$
\mathscr{C}^{+} \cap \Lambda=\left\{n G_{1}+m G_{2}: 5 n^{2}-m^{2}>0, n>0\right\},
$$


i.e., $n>0$ and $-n \sqrt{5}<m<n \sqrt{5}$. The condition $E_{11} \cdot\left(n G_{1}+m G_{2}\right) \geq 0$ gives $m \leq 0$. To get a second inequality on $n$ and $m$, let

$$
E^{\prime}=5 H-4 E_{11}-\sum_{i=1}^{10} E_{i} .
$$

Then $\left(E^{\prime}\right)^{2}=E^{\prime} \cdot K_{Y}=-1$, and $H \cdot E^{\prime}>0$. Hence, $E^{\prime}$ is effective. (In fact, one can show that $E^{\prime}$ is generically the class of an exceptional curve.) Thus, for all $n G_{1}+m G_{2} \in \bar{A}_{\text {gen }}$,

$$
E^{\prime} \cdot\left(n G_{1}+m G_{2}\right)=20 n+9 m \geq 0 ;
$$

hence,

$$
\overline{\mathscr{A}}_{\text {gen }} \cap \Lambda \subseteq\left\{n G_{1}+m G_{2}: n>0,-\frac{20}{9} n \leq m \leq 0\right\} .
$$

Next we describe the classes $\beta \in \Lambda$ with $\beta^{2}=-2$. The element $\beta=a G_{1}+b G_{2} \in \Lambda$ satisfies $\beta^{2}=-2$ if and only if $5 a^{2}-b^{2}=-1$, i.e., if and only if $b+a \sqrt{5}$ is a unit in the (nonintegrally closed) ring $\mathbb{Z}[\sqrt{5}]$. For example, the class $G_{2}$ corresponds to 1 ; as we have seen, the wall $W^{G_{2}}=W^{E_{11}}$. The fundamental unit in $\mathbb{Z}[\sqrt{5}]$ is easily checked to be $9+4 \sqrt{5}$. However, since we are only concerned with walls that are rays in the fourth quadrant $\left\{\left(n G_{1}+m G_{2}\right): n>0, m<0\right\}$, we shall consider instead $\pm(9-4 \sqrt{5})$ and shall choose the sign corresponding to $\beta=4 G_{1}-9 G_{2}$. Note that

$$
\beta \cdot\left(n G_{1}+m G_{2}\right)=40 n+18 m=0 \Longleftrightarrow E^{\prime} \cdot\left(n G_{1}+m G_{2}\right)=0 .
$$

Hence, $W^{\beta}=W^{E^{\prime}}$. Moreover, for every $\gamma \in \Lambda$ such that $\gamma^{2}=-2$ and such that the wall $W^{\gamma}$ passes through the fourth quadrant, either $W^{\gamma}=W^{\beta}$ or the corresponding ray $W^{\gamma}$ lies below $W^{\beta}$. Thus, for every $\gamma \in \Lambda$ with $\gamma^{2}=-2, r_{\gamma}$ does not preserve $\bar{A}_{\text {gen }} \cap \Lambda$. Hence, $R=\varnothing$.

Note that, aside from the isometries $r_{\beta}$, where $\beta^{2}=-2$, one can also construct isometries of infinite order preserving $\mathscr{C}^{+}$and the classes $\left[D_{i}\right]$ that do not preserve $\bar{A}_{\text {gen }}$ using multiplication by fundamental units in $\mathbb{Z}[\sqrt{5}]$ as in the previous example.

Remark 4.5. The exceptional curve $E^{\prime}$ used in the above example is part of a general series of such. For $n \geq 0$, let $Y$ be the blowup of $\mathbb{P}^{2}$ at $2 n+1$ points $p_{0}, \ldots, p_{2 n}$ with corresponding exceptional curves $E_{0}, \ldots, E_{2 n}$, and consider the divisor

$$
A=n H-(n-1) E_{0}-\sum_{i=1}^{2 n} E_{i} .
$$

Then $A^{2}=A \cdot K_{Y}=-1$, and it is easy to see that there exist $p_{0}, \ldots, p_{2 n}$ such that $A$ is the class of an exceptional curve. In fact, if $\mathbb{F}_{1}$ is the blowup of $\mathbb{P}^{2}$ at $p_{0}$, then $\Sigma=n H-(n-1) E_{0}$ is very ample on $\mathbb{F}_{1}$ and, for an anticanonical divisor $D \in\left|-K_{\mathbb{F}_{1}}\right|=\left|3 H-E_{0}\right|, \Sigma \cdot D=2 n+1$. From this, it is easy to see that we 
can choose the points $p_{1}, \ldots, p_{2 n}$ to lie on the image of $D$ in $\mathbb{P}^{2}$, and hence, we can arrange the blowup $Y$ to have (for example) an irreducible anticanonical nodal curve.

\section{Acknowledgements}

It is a pleasure to thank Mark Gross, Paul Hacking, and Sean Keel for access to their manuscript [Gross et al. 2013] and for extremely stimulating correspondence and conversations about these and other matters and Radu Laza for many helpful discussions.

\section{References}

[Bourbaki 1981] N. Bourbaki, Groupes et algèbres de Lie, Chapter 4, 5 et 6, Masson, Paris, 1981. MR 83g:17001 Zbl 0483.22001

[Coxeter 1973] H. S. M. Coxeter, Regular polytopes, 3rd ed., Dover, New York, 1973. MR 51 \#6554 Zbl 0118.35902

[Demazure 1980a] M. Demazure, "Surfaces de Del Pezzo, II: Éclater $n$ points dans $P^{2}$ ", pp. 23-35 in Séminaire sur les singularités des surfaces (Palaiseau, 1976-1977), edited by M. Demazure et al., Lecture Notes in Mathematics 777, Springer, Berlin, 1980. MR 82d:14021 Zbl 0444.14024

[Demazure 1980b] M. Demazure, "Surfaces de Del Pezzo, III: Positions presque générales", pp. 36-49 in Séminaire sur les singularités des surfaces (Palaiseau, 1976-1977), edited by M. Demazure et al., Lecture Notes in Mathematics 777, Springer, Berlin, 1980. MR 82d:14021 Zbl 0444.14024

[Demazure 1980c] M. Demazure, "Surfaces de Del Pezzo, IV: Systèmes anticanoniques", pp. 50-60 in Séminaire sur les singularités des surfaces (Palaiseau, 1976-1977), edited by M. Demazure et al., Lecture Notes in Mathematics 777, Springer, Berlin, 1980. MR 82d:14021 Zbl 0444.14024

[Demazure 1980d] M. Demazure, "Surfaces de Del Pezzo, V: Modèles anticanoniques", pp. 61-69 in Séminaire sur les singularités des surfaces (Palaiseau, 1976-1977), edited by M. Demazure et al., Lecture Notes in Mathematics 777, Springer, Berlin, 1980. MR 82d:14021 Zbl 0444.14024

[Du Val 1934] P. Du Val, "On isolated singularities of surfaces which do not affect the conditions of adjunction, II”, Proc. Camb. Philos. Soc. 30:4 (1934), 460-465. Zbl 0010.17603

[Du Val 1937] P. Du Val, “On the Kantor group of a set of points in a plane”, Proc. London Math. Soc. (2) 42:1 (1937), 18-51. MR 1577027 Zbl 0015.20302

[Friedman 1983] R. Friedman, "Linear systems on anticanonical pairs", pp. 162-171 in The birational geometry of degenerations, edited by R. Friedman and D. Morrison, Progress in Mathematics 29, Birkhäuser, Boston, 1983. Appendix to a paper of N. I. Shepherd-Barron.

[Friedman 1984] R. Friedman, “The mixed Hodge structure of an open variety”, 1984. Unpublished manuscript.

[Friedman and Miranda 1983] R. Friedman and R. Miranda, "Smoothing cusp singularities of small length”, Math. Ann. 263:2 (1983), 185-212. MR 85c:14003 Zbl 0488.14006

[Friedman and Morgan 1988] R. Friedman and J. W. Morgan, "On the diffeomorphism types of certain algebraic surfaces, I”, J. Differential Geom. 27:2 (1988), 297-369. MR 89d:57046 Zbl 0669.57016

[Friedman and Scattone 1986] R. Friedman and F. Scattone, "Type III degenerations of K3 surfaces", Invent. Math. 83:1 (1986), 1-39. MR 87k:14044 Zbl 0613.14030 
[Gross et al. 2013] M. Gross, P. Hacking, and S. Keel, "Moduli of surfaces with an anti-canonical cycle”, preprint, 2013. arXiv 1211.6367

[Looijenga 1980] E. Looijenga, "Invariant theory for generalized root systems", Invent. Math. 61:1 (1980), 1-32. MR 82f:17011 Zbl 0436.17005

[Looijenga 1981] E. Looijenga, "Rational surfaces with an anticanonical cycle", Ann. of Math. (2) 114:2 (1981), 267-322. MR 83j:14030 Zbl 0509.14035

[Manin 1986] Y. I. Manin, Cubic forms: algebra, geometry, arithmetic, 2nd ed., North-Holland Mathematical Library 4, North-Holland, Amsterdam, 1986. MR 87d:11037 Zbl 0582.14010

[Miranda and Persson 1986] R. Miranda and U. Persson, "On extremal rational elliptic surfaces", Math. Z. 193:4 (1986), 537-558. MR 88a:14044 Zbl 0652.14003

Communicated by Ravi Vakil

Received 2012-08-02 Revised 2012-11-27 Accepted 2013-01-03

rf@math.columbia.edu Department of Mathematics, Columbia University, 2990 Broadway, New York, NY, 10027, United States 


\section{Algebra \& Number Theory}

msp.org/ant

\section{EDITORS}

MANAGING EDITOR

Bjorn Poonen

Massachusetts Institute of Technology

Cambridge, USA

\author{
EDITORIAL BOARD CHAIR \\ David Eisenbud \\ University of California \\ Berkeley, USA
}

\section{BOARD OF EDITORS}

Georgia Benkart

Dave Benson

Richard E. Borcherds

John H. Coates

J-L. Colliot-Thélène

Brian D. Conrad

Hélène Esnault

Hubert Flenner

Edward Frenkel

Andrew Granville

Joseph Gubeladze

Ehud Hrushovski

Craig Huneke

Mikhail Kapranov

Yujiro Kawamata

János Kollár

Yuri Manin

Barry Mazur

Philippe Michel
University of Wisconsin, Madison, USA

University of Aberdeen, Scotland

University of California, Berkeley, USA

University of Cambridge, UK

CNRS, Université Paris-Sud, France

University of Michigan, USA

Freie Universität Berlin, Germany

Ruhr-Universität, Germany

University of California, Berkeley, USA

Université de Montréal, Canada

San Francisco State University, USA

Hebrew University, Israel

University of Virginia, USA

Yale University, USA

University of Tokyo, Japan

Princeton University, USA

Northwestern University, USA

Harvard University, USA

École Polytechnique Fédérale de Lausanne
Susan Montgomery

Shigefumi Mori

Raman Parimala

Jonathan Pila

Victor Reiner

Karl Rubin

Peter Sarnak

Joseph H. Silverman

Michael Singer

Vasudevan Srinivas

J. Toby Stafford

Bernd Sturmfels

Richard Taylor

Ravi Vakil

Michel van den Bergh

Marie-France Vignéras

Kei-Ichi Watanabe

Efim Zelmanov
University of Southern California, USA

RIMS, Kyoto University, Japan

Emory University, USA

University of Oxford, UK

University of Minnesota, USA

University of California, Irvine, USA

Princeton University, USA

Brown University, USA

North Carolina State University, USA

Tata Inst. of Fund. Research, India

University of Michigan, USA

University of California, Berkeley, USA

Harvard University, USA

Stanford University, USA

Hasselt University, Belgium

Université Paris VII, France

Nihon University, Japan

University of California, San Diego, USA

\section{PRODUCTION}

production@msp.org

Silvio Levy, Scientific Editor

See inside back cover or msp.org/ant for submission instructions.

The subscription price for 2013 is US \$200/year for the electronic version, and \$350/year ( $\$ 40$, if shipping outside the US) for print and electronic. Subscriptions, requests for back issues and changes of subscribers address should be sent to MSP.

Algebra \& Number Theory (ISSN 1944-7833 electronic, 1937-0652 printed) at Mathematical Sciences Publishers, 798 Evans Hall \#3840, c/o University of California, Berkeley, CA 94720-3840 is published continuously online. Periodical rate postage paid at Berkeley, CA 94704, and additional mailing offices.

ANT peer review and production are managed by EditFLOW ${ }^{\circledR}$ from Mathematical Sciences Publishers.

\section{PUBLISHED BY}

- mathematical sciences publishers

nonprofit scientific publishing

http://msp.org/

(C) 2013 Mathematical Sciences Publishers 


\section{Algebra \& Number Theory}

Volume $7 \quad$ No. $6 \quad 2013$

On the discrete logarithm problem in elliptic curves II

Claus Diem

Identifying Frobenius elements in Galois groups

1325

TIM DOKCHITSER and VLADIMIR DOKCHITSER

Weak approximation for cubic hypersurfaces of large dimension

1353

MIKE SWARBRICK JONES

The Picard crossed module of a braided tensor category

1365

ALEXEI DAVYDOV and DMITRI NIKSHYCH

A Gross-Zagier formula for quaternion algebras over totally real fields

1405

EYAL Z. GOREN and KRISTIN E. LAUTER

Counting rational points over number fields on a singular cubic surface

CHRISTOPHER FREI

On the ample cone of a rational surface with an anticanonical cycle

ROBERT FRIEDMAN

Commuting involutions of Lie algebras, commuting varieties, and simple Jordan algebras

DMITRI I. PANYUSHEV 